\title{
One-Pot, Three-Component Synthesis of Linear-Substituted Homoallylic Alcohols via Allyl(isopropoxy)dimethylsilane
}

\author{
Lianhai Li ${ }^{*}$ and Neenah Navasero \\ Department of Medicinal Chemistry, Merck Frosst Centre for \\ Therapeutic Research \\ PO Box 1005, Pointe-Claire-Dorval, Quebec, Canada H9R 4P8. \\ Lianhaili@merck.com
}

\section{General Experimental}

Unless otherwise stated, reactions were performed under a nitrogen atmosphere using commercially obtained anhydrous solvents as received. Alkyllithium reagents were titrated with 1,10-phenanthroline as an indicator in ether. ${ }^{1}$ All other commercially obtained reagents were used as received. All reactions were magnetically stirred and monitored by thin-layer chromatography (TLC) using E. Merck silica gel 60 F254 precoated plates $(0.25-\mathrm{mm})$. Column or flash chromatography was performed with the indicated solvents using silica gel (particle size 0.032-0.063 $\mathrm{nm}$ ) purchased from Bodman. ${ }^{1} \mathrm{H}$ and ${ }^{13} \mathrm{C}$ NMR were recorded on Bruker Advance DPX-500 using acetone- $d_{6}$ as the solvent. Chemical shifts $(\delta)$ are given in parts per million (ppm) relative to internal solvent (acetone-d6: ${ }^{1} \mathrm{H} @ 2.05$ ppm, ${ }^{13} \mathrm{C} @ 29.84$ ppm), and coupling constants $(J)$ are given in hertz $(\mathrm{Hz})$.

\section{Experimental:}

Allyl(isopropoxy)dimethylsilane (6): ${ }^{2}$

\footnotetext{
${ }^{1}$ Stanetty, P.; Mihovilovic, M.D. J. Org. Chem. 1997, 62, 1514-1515.

${ }^{2}$ Tamao, K.; Nakajo, E.; Ito, Y. Synthetic Commun. 1987, 17, 1637.
} 


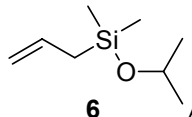

$6 \quad$ Allylchlorodimethylsilane (13.47 g, $0.1 \mathrm{~mol})$ was added dropwise to a solution of $i$-PrOH and imidazole $(16.3 \mathrm{~g})$ in $150 \mathrm{~mL}$ of DMF stirred at $0{ }^{\circ} \mathrm{C}$. The resulting mixture was stirred at room temperature for $3 \mathrm{hr}$ after the addition. It was poured into a separatory funnel containing pentane $(300 \mathrm{~mL})$ and $\mathrm{H}_{2} \mathrm{O}(300 \mathrm{~mL})$. The organic layer was separated and washed with brine, dried with anhydrous sodium sulphate. The solvent was removed by careful distillation and the residue was purified by distillation (bp 131-2 $\left.{ }^{\circ} \mathrm{C}, \mathrm{d}=0.783 \mathrm{~g} / \mathrm{mL}\right)$ to afford a clear oil (15.6 g, 99\% yield). ${ }^{1} \mathrm{H}$ NMR (500 MHz, Acetone-d6): $\delta$ 5.84-5.76 (m, $1 \mathrm{H}) ; 4.89-4.79(\mathrm{~m}, 2 \mathrm{H}) ; 4.06-3.99$ (m, $1 \mathrm{H}) ; 1.58(\mathrm{~d}$, $J=7.1 \mathrm{~Hz}, 2 \mathrm{H}) ; 1.10(\mathrm{~d}, J=6.0 \mathrm{~Hz}, 6 \mathrm{H}) ; 0.07$ (s, $6 \mathrm{H}) .{ }^{13} \mathrm{C} \mathrm{NMR}(126 \mathrm{MHz}$, Acetone-d6): $\delta 135.28,113.62,65.42,26.13,25.48,-1.83$.

(3E)-4-[isopropoxy(dimethyl)silyl]-1-phenylbut-3-en-1-ol (7). ${ }^{3}$<smiles>CC(C)O[Si](C)(C)/C=C/CC(O)c1ccccc1</smiles>

7 To a solution of allyl(isopropoxy)dimethylsilane (6) (1.01 mL, 5.0 $\mathrm{mmol})$ in $20 \mathrm{~mL}$ of THF at $-78^{\circ} \mathrm{C}$ under a nitrogen atmosphere, $s$-BuLi in cyclohexane (5.5 mmol, 1.1 equiv) was added dropwise. Having been stirred for $0.5 \mathrm{hr}$ at $-78{ }^{\circ} \mathrm{C}$ after the addition, benzaldehyde ( $0.61 \mathrm{~mL}, 6 \mathrm{mmol}, 1.2$ equiv) was added dropwise, and the resulting mixture was further stirred for $0.5 \mathrm{hr}$ at $-78^{\circ} \mathrm{C}$. The reaction was quenched by the addition of saturated $\mathrm{NaHCO}_{3}$ and extracted with EtOAc/Hexanes (3:7), dried with $\mathrm{Na}_{2} \mathrm{SO}_{4}$, filtered, and evaporated. By addition of $2 \mathrm{mmol}$ of toluene to the crude as internal standard, the yield of the 7 was determined by ${ }^{1} \mathrm{H}$ NMR to be $70 \%$. Compound 7 was obtained in pure form as a clear oil ( $0.86 \mathrm{~g}, 65 \%$ yield) by flash chromatography over florisil ${ }^{4}$ (5-10\% EtOAc/hexanes). ${ }^{1} \mathrm{H}$ NMR (500 MHz, acetone- $\left.d 6\right): \delta 7.36(\mathrm{~d}, J=$ $7.2 \mathrm{~Hz}, 2 \mathrm{H}) ; 7.30(\mathrm{t}, J=7.2 \mathrm{~Hz}, 2 \mathrm{H}) ; 7.20(\mathrm{t}, J=7.2 \mathrm{~Hz}, 1 \mathrm{H}) ; 6.20$ (dt, $J=18.7,6.6$ $\mathrm{Hz}, 1 \mathrm{H}) ; 5.64(\mathrm{~d}, J=18.8,1 \mathrm{H}) ; 4.75(\mathrm{dt}, J=4.1,7.0 \mathrm{~Hz}, 1 \mathrm{H}, 1 \mathrm{H}) ; 4.25(\mathrm{~d}, J=4.1$ $\mathrm{Hz}, 1 \mathrm{H}, \mathrm{OH}) ; 3.94-3.88(\mathrm{~m}, 1 \mathrm{H}) ; 2.57-2.49(\mathrm{~m}, 2 \mathrm{H}) ; 1.05(\mathrm{~d}, J=6.0 \mathrm{~Hz}, 6 \mathrm{H}) ; 0.07$ $(\mathrm{s}, 6 \mathrm{H}) .{ }^{13} \mathrm{C}$ NMR $(126 \mathrm{MHz}$, Acetone-d6): $\delta 146.38,146.30,131.56,128.76,127.62$,

\footnotetext{
${ }^{3}$ Tamao, K.; Nakajo, E.; Ito, Y. Tetrahedron 1988, 44, 3997.

${ }^{4}$ Compound 7 is not stable when purified by silica gel column even after pre-treatment with triethylamine.
} 
126.74, 73.80, 65.29, 47.88, 26.12, -0.95, -0.97. HRMS (FAB $\left.{ }^{+}\right)$: Calculated for $\mathrm{C}_{15} \mathrm{H}_{24} \mathrm{O}_{2} \mathrm{Si}+\mathrm{K}^{+}$: 303.1183; Found: 303.1183 .

\section{General Procedure for Pd-catalyzed Cross-coupling of 7 with Aryl/Vinyl Halide:}

To a mixture of (3E)-4-[isopropoxy(dimethyl)silyl]-1-phenylbut-3-en-1-ol (7) (212 mg, $0.8 \mathrm{mmol})$, aryl/vinyl halide $\left(0.8 \mathrm{mmol}, 1\right.$ equiv), and $\left(\operatorname{Pd}_{2}(\mathrm{dba})_{3}\right.$ or $(\text { allylPdCl})_{2}$ (0.04 mmol, 0.05 equiv) in $5 \mathrm{~mL}$ of THF under a $\mathrm{N}_{2}$ atmosphere, $1.6 \mathrm{~mL}$ of TBAF in THF ( $1 \mathrm{M}, 1.6 \mathrm{mmol}, 2$ equiv) was added. The resulting mixture was stirred for certain period of time as indicated in Table 2 at $\mathrm{rt}$ for aryl iodide or at $50{ }^{\circ} \mathrm{C}$ for aryl/vinyl bromide. The reaction was quenched by the addition of water and extracted with EtOAc (15 mL $\times 3$ ). The combined organic phase was then dried with $\mathrm{Na}_{2} \mathrm{SO}_{4}$ and evaporated. All compounds were purified by flash chromatography.

(3E)-1,4-diphenylbut-3-en-1-ol (1a). ${ }^{5}$

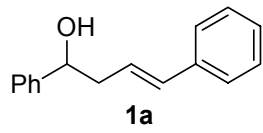

7 with iodobenzene $(0.8 \mathrm{mmol})$ at $\mathrm{rt}$ afforded $1 \mathrm{a}$ as a white solid (174 $\mathrm{mg}, 97 \%$ yield);

Following the above procedure with (allylPdCl $)_{2}$ as catalyst, the coupling of 7 with bromobenzene $(0.8 \mathrm{mmol})$ at $50{ }^{\circ} \mathrm{C}$ afforded $1 \mathrm{a}$ as a white solid $(145 \mathrm{mg}, 82 \%$ yield $) .{ }^{1} \mathrm{H}$ NMR (500 MHz, Acetone-d6): $\delta 7.41$ (d, $J=7.3$ Hz, $2 \mathrm{H})$; 7.34-7.26 (m, $6 \mathrm{H})$; 7.23$7.16(\mathrm{~m}, 2 \mathrm{H}) ; 6.43(\mathrm{~d}, J=15.9 \mathrm{~Hz}, 1 \mathrm{H}) ; 6.31(\mathrm{dt}, J=15.9,7.1 \mathrm{~Hz}, 1 \mathrm{H}) ; 4.79$ (dt, $J=$ $4.1,7.0 \mathrm{~Hz}, 1 \mathrm{H}) ; 4.35(\mathrm{~d}, J=4.1 \mathrm{~Hz}, 1 \mathrm{H}) ; 2.60(\mathrm{dd}, J=7.0,7.1 \mathrm{~Hz}, 2 \mathrm{H}) .{ }^{13} \mathrm{C} \mathrm{NMR}$ (126 MHz, Acetone-d6): $\delta$ 146.47, 138.66, 132.74, 129.30, 128.85, 128.04, 127.76, 127.72, 126.79, 126.72, 74.17, 44.27. HRMS (FAB $\left.{ }^{+}\right)$: Calculated for $\mathrm{C}_{16} \mathrm{H}_{16} \mathrm{O}+\mathrm{K}^{+}$: 263.0838; Found: 263.0838.

Ethyl 4-[(1E)-4-hydroxy-4-phenylbut-1-en-1-yl]benzoate (1b).

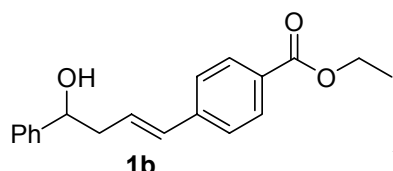
coupling of 7 with ethyl-4-iodobenzoate $(0.8 \mathrm{mmol})$ at $\mathrm{rt}$ afforded $\mathbf{1 b}$ as a white solid

\footnotetext{
${ }^{5}$ Tan, K.-T; Chng, S.-S. ; Cheng, H.-S. ; Loh,, T.-P. J. Am. Chem. Soc. 2003, 125, 2858.
} 
(233 mg, 98\% yield). ${ }^{1} \mathrm{H}$ NMR (500 MHz, acetone- $\left.d 6\right): \delta 7.93(\mathrm{~d}, J=8.3 \mathrm{~Hz}, 2 \mathrm{H}) ; 7.47$ $(\mathrm{d}, J=8.3 \mathrm{~Hz}, 2 \mathrm{H}) ; 7.41(\mathrm{~d}, J=7.4 \mathrm{~Hz}, 2 \mathrm{H}) ; 7.32(\mathrm{t}, J=7.6 \mathrm{~Hz}, 2 \mathrm{H}) ; 7.22(\mathrm{t}, J=7.3$ $\mathrm{Hz}, 1 \mathrm{H})$; 6.53-6.49 (m, $2 \mathrm{H}) ; 4.83(\mathrm{dt}, J=4.1,6.2 \mathrm{~Hz}, 1 \mathrm{H}) ; 4.42(\mathrm{~d}, J=4.1 \mathrm{~Hz}, 1 \mathrm{H}$ $\mathrm{OH}) ; 4.31(\mathrm{q}, J=7.1 \mathrm{~Hz}, 2 \mathrm{H}) ; 2.65(\mathrm{~m}, 2 \mathrm{H}) ; 1.34(\mathrm{t}, J=7.1 \mathrm{~Hz}, 3 \mathrm{H}) .{ }^{13} \mathrm{C} \mathrm{NMR}$ (126 MHz, acetone-d6): $\delta 166.28,146.15,142.96,131.65,131.14,130.24,129.48$, 128.67, 127.52, 126.54, 126.47, 73.75, 61.07, 44.07, 14.36. HRMS (FAB ${ }^{+}$): Calculated for $\mathrm{C}_{19} \mathrm{H}_{20} \mathrm{O}_{3}+\mathrm{H}^{+}$: 297.1490; Found: 297.1491.

1-\{4-[(1E)-4-hydroxy-4-phenylbut-1-en-1-yl]phenyl $\}$ ethanone (1c).

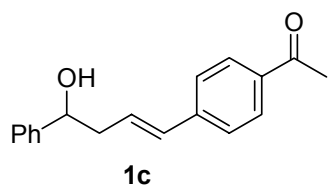
coupling of 7 with 4-iodoacetophenone $(0.8 \mathrm{mmol})$ at $\mathrm{rt}$ afforded $1 \mathrm{c}$ as a white solid (205 mg, 96\% yield). ${ }^{1} \mathrm{H}$ NMR (500 MHz, acetone- $\left.d 6\right): \delta 7.91(\mathrm{~d}, J=8.5 \mathrm{~Hz}, 2 \mathrm{H}) ; 7.47$ (d, $J$ $=8.4 \mathrm{~Hz}, 2 \mathrm{H}) ; 7.41(\mathrm{~d}, J=7.2 \mathrm{~Hz}, 2 \mathrm{H}) ; 7.32(\mathrm{t}, J=7.6 \mathrm{~Hz}, 2 \mathrm{H}) ; 7.22(\mathrm{t}, J=7.3 \mathrm{~Hz}, 1$ $\mathrm{H}) ; 6.51(\mathrm{~m}, 2 \mathrm{H}) ; 4.83(\mathrm{dt}, J=4.1,4.7 \mathrm{~Hz}, 1 \mathrm{H}) ; 4.41(\mathrm{~d}, J=4.1 \mathrm{~Hz}, 1 \mathrm{H} \mathrm{OH}) ; 2.66-$ $2.62(\mathrm{~m}, 2 \mathrm{H}) ; 2.54$ (s, $3 \mathrm{H}) .{ }^{13} \mathrm{C}$ NMR (126 MHz, acetone-d6): $\delta 197.29,146.36,143.13$, 136.60, 131.86, 131.40, 129.44, 128.86, 127.71, 126.80, 126.67, 73.94, 44.29, 26.57.

HRMS (FAB ${ }^{+}$): Calculated for $\mathrm{C}_{18} \mathrm{H}_{18} \mathrm{O}_{2}+\mathrm{H}^{+}$: 267.1386; Found: 267.1385.

(3E)-4-(4-methoxyphenyl)-1-phenylbut-3-en-1-ol (1d). ${ }^{6}$

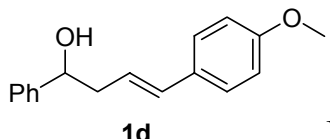

Following the above procedure with $\left(\mathrm{Pd}_{2}(\mathrm{dba})_{3}\right.$ as catalyst, the coupling of 7 with 4-iodoanisole $(0.8 \mathrm{mmol})$ at $\mathrm{rt}$ afforded $\mathbf{1 d}$ as a white solid $(184 \mathrm{mg}$, 90\% yield). ${ }^{1} \mathrm{H}$ NMR (500 MHz, acetone- $\left.d 6\right): \delta 7.40(\mathrm{~d}, J=7.2 \mathrm{~Hz}, 2 \mathrm{H}) ; 7.30(\mathrm{t}, J=$ $7.2 \mathrm{~Hz}, 2 \mathrm{H}) ; 7.26(\mathrm{~d}, J=8.7 \mathrm{~Hz}, 2 \mathrm{H}) ; 7.21(\mathrm{t}, J=7.3 \mathrm{~Hz}, 1 \mathrm{H}) ; 6.84(\mathrm{~d}, J=8.7 \mathrm{~Hz}, 2$ $\mathrm{H}) ; 6.36(\mathrm{~d}, J=15.9 \mathrm{~Hz}, 1 \mathrm{H}) ; 6.14(\mathrm{dt}, J=15.9,7.2 \mathrm{~Hz}, 1 \mathrm{H}) ; 4.76(\mathrm{dt}, J=4.1,5.8 \mathrm{~Hz}$, $1 \mathrm{H}) ; 4.30(\mathrm{~d}, J=4.1 \mathrm{~Hz}, 1 \mathrm{H}, \mathrm{OH}) ; 3.76(\mathrm{~s}, 3 \mathrm{H}) ; 2.59-2.52(\mathrm{~m}, 2 \mathrm{H}) .{ }^{13} \mathrm{C} \mathrm{NMR}(126$ MHz, acetone- $d 6): \delta 159.85,146.55,132.20,131.33,128.80,127.90,127.61,126.71$, 125.53, 114.65, 74.29, 55.44, 44.30. $\mathrm{HRMS}\left(\mathrm{FAB}^{+}\right)$: Calculated for $\mathrm{C}_{17} \mathrm{H}_{18} \mathrm{O}+\mathrm{K}^{+}$: 293.0943; Found: 293.0944.

\footnotetext{
${ }^{6}$ Materal-Saadi, M. S. Phosphorus, Sulfur Silicon Relat Elem 2000, 164, 269.
} 
(3E)-4-(2-methylphenyl)-1-phenylbut-3-en-1-ol (1e).

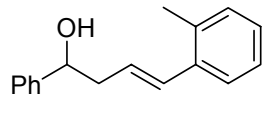

1e Following the above procedure with $\left(\mathrm{Pd}_{2}(\mathrm{dba})_{3}\right.$ as catalyst, the coupling of 7 with 2-iodotoluene $(0.8 \mathrm{mmol})$ at $\mathrm{rt}$ afforded $1 \mathrm{e}$ as a white solid (153 $\mathrm{mg}, 80 \%$ yield); Following the above procedure with (allylPdCl $)_{2}$ as catalyst, the coupling of 7 with 2 iodotoluene $(0.8 \mathrm{mmol})$ at $\mathrm{rt}$ afforded $1 \mathrm{e}$ as a white solid $(188 \mathrm{mg}, 99 \%$ yield $) .{ }^{1} \mathrm{H}$ NMR (500 MHz, acetone- $d 6): \delta 7.41(\mathrm{~d}, J=7.1 \mathrm{~Hz}, 2 \mathrm{H}) ; 7.38-7.34(\mathrm{~m}, 1 \mathrm{H}) ; 7.31(\mathrm{t}, J=7.6$ $\mathrm{Hz}, 2 \mathrm{H}) ; 7.21$ (t, $J=7.3 \mathrm{~Hz}, 1 \mathrm{H}) ; 7.10-7.08(\mathrm{~m}, 3 \mathrm{H}) ; 6.60$ (d, $J=15.7 \mathrm{~Hz}, 1 \mathrm{H}) ; 6.13$ $(\mathrm{dt}, J=15.7,7.2 \mathrm{~Hz}, 1 \mathrm{H}) ; 4.81(\mathrm{dt}, J=4.1,6.5 \mathrm{~Hz}, 1 \mathrm{H}) ; 4.38(\mathrm{~d}, J=4.1 \mathrm{~Hz}, 1 \mathrm{H}, \mathrm{OH})$; 2.65-2.63 (m, $2 \mathrm{H}) ; 2.23(\mathrm{~s}, 3 \mathrm{H}) .{ }^{13} \mathrm{C}$ NMR (126 MHz, acetone-d6): $\delta 130.84,130.68$, 129.22, 128.80, 127.70, 127.63, 126.78, 126.19, 74.20, 44.42, 19.74. HRMS (FAB $\left.{ }^{+}\right)$: Calculated for $\mathrm{C}_{17} \mathrm{H}_{18} \mathrm{O}+\mathrm{H}^{+}-\mathrm{H}_{2} \mathrm{O}$ : 221.1329; Found: 221.1330 .

(3E)-4-(2-methoxyphenyl)-1-phenylbut-3-en-1-ol (1f).<smiles>COc1ccccc1/C=C/CC(O)c1ccccc1</smiles>

1f Following the above procedure with (allylPdCl $)_{2}$ as catalyst, the coupling of 7 with 2-iodoanisole ( $0.8 \mathrm{mmol})$ at $\mathrm{rt}$ afforded $\mathbf{1 f}$ as a clear oil (200 $\mathrm{mg}, 93 \%$ yield). ${ }^{1} \mathrm{H}$ NMR (500 MHz, acetone- $\left.d 6\right): \delta 7.40(\mathrm{t}, J=9.7 \mathrm{~Hz}, 3 \mathrm{H}) ; 7.31(\mathrm{t}, J=7.6 \mathrm{~Hz}, 2 \mathrm{H})$; $7.22-7.15(\mathrm{~m}, 2 \mathrm{H}) ; 6.93(\mathrm{~d}, J=8.1 \mathrm{~Hz}, 1 \mathrm{H}) ; 6.86(\mathrm{t}, J=7.4 \mathrm{~Hz}, 1 \mathrm{H}) ; 6.71(\mathrm{~d}, J=15.8$ $\mathrm{Hz}, 1 \mathrm{H}) ; 6.26(\mathrm{dt}, J=15.8,7.2 \mathrm{~Hz}, 1 \mathrm{H}) ; 4.78(\mathrm{dt}, J=4.2,5.8 \mathrm{~Hz}, 1 \mathrm{H}) ; 4.31(\mathrm{~d}, J=$ $4.2 \mathrm{~Hz}, 1 \mathrm{H} \mathrm{OH}) ; 3.80(\mathrm{~s}, 3 \mathrm{H}) ; 2.63-2.59$ (m, $2 \mathrm{H}) .{ }^{13} \mathrm{C}$ NMR (126 MHz, acetone-d6): $\delta 157.25,146.55,128.91,128.81,128.19,127.61,127.31,126.91,126.73,121.27,111.76$, 74.29, 55.67, 44.76. HRMS (FAB ${ }^{+}$): Calculated for $\mathrm{C}_{17} \mathrm{H}_{18} \mathrm{O}_{2}+\mathrm{K}^{+}$: 293.0943; Found: 293.0944.

(3E)-5-methyl-1-phenylhexa-3,5-dien-1-ol (1g).<smiles>C=C(C)C=CCC(O)c1ccccc1</smiles>

$1 \mathrm{~g}$ Following the above procedure with (allylPdCl $)_{2}$ as catalyst, the coupling of 7 with 2-bromopropene $(0.8 \mathrm{mmol})$ at $50{ }^{\circ} \mathrm{C}$ afforded $1 \mathrm{~g}$ was afforded as a clear oil (57 
mg, 38\% yield). ${ }^{7}{ }^{1} \mathrm{H}$ NMR (500 MHz, acetone- $\left.d 6\right): \delta 7.36(\mathrm{~m}, 2 \mathrm{H}) ; 7.30(\mathrm{~m}, 2 \mathrm{H}) ; 7.21$ $(\mathrm{t}, J=7.2 \mathrm{~Hz}, 1 \mathrm{H}) ; 6.17(\mathrm{~d}, J=15.6 \mathrm{~Hz}, 1 \mathrm{H}) ; 5.78-5.70(\mathrm{dt}, J=15.6,6.8 \mathrm{~Hz}, 1 \mathrm{H})$; $4.85(\mathrm{~d}, J=6.0 \mathrm{~Hz}, 2 \mathrm{H}) ; 4.74-4.70(\mathrm{~m}, 1 \mathrm{H}) ; 4.31(\mathrm{~d}, J=4.1 \mathrm{~Hz}, 1 \mathrm{H}, \mathrm{OH}) ; 2.54-2.46$ $(\mathrm{m}, 2 \mathrm{H}) ; 1.77(\mathrm{~s}, 3 \mathrm{H})$.

(3E,5E)-1,6-diphenylhexa-3,5-dien-1-ol (1h).<smiles>OC(C/C=C/C=C/c1ccccc1)c1ccccc1</smiles>

1h Following the above procedure with (allylPdCl $)_{2}$ as catalyst, the coupling of 7 with $\beta$-bromostyrene $(E / Z=6: 1,0.8 \mathrm{mmol})$ at $50{ }^{\circ} \mathrm{C}$ afforded $\mathbf{1 h}$ as a clear oil (132 mg, 66\% yield) with about 6:1 ratio of $(E, E)$-isomer to $(E, Z)$-isomer. ${ }^{8}$ Major isomer: ${ }^{1} \mathrm{H}$ NMR (500 MHz, acetone- $\left.d 6\right): \delta$ 7.55-7.20 (m, $\left.10 \mathrm{H}\right) ; 6.83(\mathrm{dd}, J=15.7,10.4$ $\mathrm{Hz}, 1 \mathrm{H}) ; 6.47(\mathrm{~d}, J=15.6 \mathrm{~Hz}, 1 \mathrm{H}) ; 6.25(\mathrm{dd}, J=15.4,10.6 \mathrm{~Hz}, 1 \mathrm{H}) ; 5.87$ (dt, $J=$ $15.3,7.1 \mathrm{~Hz}, 1 \mathrm{H}) ; 4.80-4.70(\mathrm{~m}, 1 \mathrm{H}) ; 4.30(\mathrm{~d}, J=4.4 \mathrm{~Hz}, 1 \mathrm{H}, \mathrm{OH}) ; 2.60-2.48(\mathrm{~m}, 2$ $\mathrm{H})$.

\section{General Procedure of One-pot Process (compounds 1a-f, 1i-o):}

To a solution of allyl(isopropoxy)dimethylsilane (6) (600 uL, $2.97 \mathrm{mmol}, 1$ equiv ) in THF $(6 \mathrm{~mL}, 0.35 \mathrm{M})$ at $-78{ }^{\circ} \mathrm{C}, 2.6 \mathrm{~mL}$ of sec-Butyllithium in cyclohexane $(1.4 \mathrm{M}, 3.64$ mmol, 1.1 equiv) was added dropwise. The resulting mixture was stirred at $-78{ }^{\circ} \mathrm{C}$ for 1 $\mathrm{hr}$ and the desired carbonyl compound (3.65 mmol, 1.1 equiv) was added dropwise. After being stirred for $0.5 \mathrm{hr}$ at $-78{ }^{\circ} \mathrm{C}$, the reaction was warmed to $0{ }^{\circ} \mathrm{C}$, and $\mathrm{AcOH}(210$ $\mathrm{uL}, 3.67 \mathrm{mmol}, 1.1$ equiv) was added dropwise. Then, aryl iodide ( $2.1 \mathrm{mmol}, 0.7$ equiv), and $\mathrm{Pd}_{2}(\mathrm{dba})_{3}(0.04 \mathrm{mmol}, 0.05 \text { equiv) or (allylPdCl})_{2}(0.04 \mathrm{mmol}, 0.05$ equiv) was added. The system was purged with $\mathrm{N}_{2}$, and then TBAF ( $1 \mathrm{M}$ in THF, $7.2 \mathrm{~mL}, 7.2 \mathrm{mmol}$, 2.4 equiv) was added. The resulting mixture was stirred at room temperature overnight. The reaction was quenched with water, extracted with EtOAc, dried over $\mathrm{Na}_{2} \mathrm{SO}_{4}$,

\footnotetext{
${ }^{7}$ Compound $1 \mathrm{~g}$ is not stable when existed in pure form; the relatively low yield might be due to its instability under the reaction conditions. No effort was done to improve the yield.

${ }^{8}$ The ratio agrees with the trans-cis ratio of $\beta$-bromostyrene used.
} 
filtered, and evaporated. The residue was purified by flash chromatography to afford the desired product.

Methyl 2-[(1E)-4-hydroxy-4-phenylbut-1-en-1-yl]benzoate (1i).<smiles>COC(=O)c1ccccc1/C=C/CC(O)c1ccccc1</smiles>
iodobenzoate in the presence of $(\text { allylPdCl })_{2}$ as catalyst, $\mathbf{1 i}$ was afforded as a clear oil in $56 \%$ yield (334 mg). ${ }^{1} \mathrm{H}$ NMR (500 MHz, acetone- $\left.d 6\right): \delta 7.79(\mathrm{~d}, J=7.9 \mathrm{~Hz}, 1 \mathrm{H}) ; 7.58$ $(\mathrm{d}, J=7.8 \mathrm{~Hz}, 1 \mathrm{H}) ; 7.49$ (t, $J=7.5 \mathrm{~Hz}, 1 \mathrm{H}) ; 7.42(\mathrm{~d}, J=7.3 \mathrm{~Hz}, 2 \mathrm{H}) ; 7.34-7.27$ (m, 3 $\mathrm{H}) ; 7.22(\mathrm{t}, J=7.3 \mathrm{~Hz}, 1 \mathrm{H}) ; 7.17(\mathrm{~d}, J=15.9 \mathrm{~Hz}, 1 \mathrm{H}) ; 6.25-6.19(\mathrm{dt}, J=15.8,7.1 \mathrm{~Hz}$, $1 \mathrm{H}) ; 4.83-4.81(\mathrm{~m}, 1 \mathrm{H}) ; 4.34(\mathrm{~d}, J=4.1 \mathrm{~Hz}, 1 \mathrm{H}, \mathrm{OH}) ; 3.82(\mathrm{~s}, 3 \mathrm{H}) ; 2.63(\mathrm{t}, J=6.8$ $\mathrm{Hz}, 2 \mathrm{H}) .{ }^{13} \mathrm{C}$ NMR $(126 \mathrm{MHz}$, acetone-d6): $\delta 168.30,146.35,139.84,132.75,131.23$, $130.89,130.86,129.43,128.87,127.77,127.70,127.60,126.73,74.08,52.26,44.38$.

(4E)-2-methyl-5-phenylpent-4-en-2-ol (1j).

$\mathrm{X}^{\mathrm{OH}} \mathrm{Ph}^{\mathrm{P}}$

1j Following the above procedure, with acetone and iodobenzene in the presence of $\mathrm{Pd}_{2}(\mathrm{dba})_{3}$ as catalyst, $\mathbf{1} \mathbf{j}$ was afforded as a clear oil in $71 \%$ yield $(260 \mathrm{mg}) .{ }^{1} \mathrm{H} \mathrm{NMR}$ (500 MHz, acetone-d6): $\delta 7.39(\mathrm{~d}, J=7.3 \mathrm{~Hz}, 2 \mathrm{H}) ; 7.29(\mathrm{t}, J=7.7 \mathrm{~Hz}, 2 \mathrm{H}) ; 7.18(\mathrm{t}, J=$ $7.3 \mathrm{~Hz}, 1 \mathrm{H}) ; 6.45-6.37$ (m, $2 \mathrm{H}) ; 3.42$ (s, $1 \mathrm{H}, \mathrm{OH}) ; 2.35$ (d, J=5.9 Hz, $2 \mathrm{H}) ; 1.20$ (s, 6 H). ${ }^{13} \mathrm{C}$ NMR (126 MHz, acetone-do): $\delta 138.87,133.09,129.40,128.23,127.80,126.93$, 70.60, 48.47, 29.80. HRMS (FAB ${ }^{+}$): Calculated for $\left[\mathrm{C}_{12} \mathrm{H}_{16} \mathrm{O}-\mathrm{H}\right]^{+}$: 175.1124; Found: 175.1123 .

1-[(2E)-3-phenylprop-2-en-1-yl]cyclohexanol (1k).

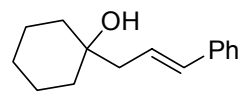

1k Following the above procedure, with cyclohexanone and iodobenzene in the presence of $\mathrm{Pd}_{2}(\mathrm{dba})_{3}$ as catalyst, $1 \mathbf{k}$ was afforded as a clear oil in $62 \%$ yield $(281 \mathrm{mg})$. ${ }^{1} \mathrm{H}$ NMR (500 MHz, acetone- $\left.d 6\right): \delta 7.39(\mathrm{~d}, J=7.3 \mathrm{~Hz}, 2 \mathrm{H}) ; 7.28(\mathrm{t}, J=7.3 \mathrm{~Hz}, 2 \mathrm{H})$; $7.18(\mathrm{t}, J=7.3 \mathrm{~Hz}, 1 \mathrm{H}) ; 6.44-6.40(\mathrm{~m}, 2 \mathrm{H}) ; 3.18(\mathrm{~s}, 1 \mathrm{H}, \mathrm{OH}) ; 2.34-2.32(\mathrm{~m}, 2 \mathrm{H})$; 
$1.69-1.61(\mathrm{~m}, 2 \mathrm{H}) ; 1.60-1.48(\mathrm{~m}, 3 \mathrm{H}) ; 1.48-1.39(\mathrm{~m}, 4 \mathrm{H}) ; 1.27-1.19(\mathrm{~m}, 1 \mathrm{H}) .{ }^{13} \mathrm{C}$ NMR (126 MHz, acetone-d6): $\delta 138.81,133.00,129.28,127.64,126.81,71.28,47.37$, 38.21, 26.65, 22.81. HRMS (FAB ${ }^{+}$): Calculated for $\mathrm{C}_{15} \mathrm{H}_{20} \mathrm{O}+\mathrm{Na}^{+}$: 239.1411; Found: 249.1412 .

(5E)-1,6-diphenylhex-5-en-3-ol (11).

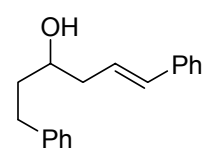

1 Following the above procedure, with 3-phenylpropanal and iodobenzene in the presence of $\mathrm{Pd}_{2}(\mathrm{dba})_{3}$ as catalyst, 11 was afforded as a white solid in $87 \%$ yield. ${ }^{1} \mathrm{H}$ NMR (500 MHz, acetone- $d 6): \delta 7.37$ (d, $J=7.4$ Hz, 2 H); 7.29-7.13 (m, 8 H); 6.45 (d, $J$ $=16.0 \mathrm{~Hz}, 1 \mathrm{H}) ; 6.36(\mathrm{dt}, J=16.0,7.0 \mathrm{~Hz} 1 \mathrm{H}) ; 3.76(\mathrm{~d}, J=5.2 \mathrm{~Hz}, 1 \mathrm{H}, \mathrm{OH}) ; 3.74-$ $3.68(\mathrm{~m}, 1 \mathrm{H}) ; 2.86-2.64(\mathrm{~m}, 2 \mathrm{H}) ; 2.43-2.35(\mathrm{~m}, 2 \mathrm{H}) ; 1.85-1.69$ (m, $2 \mathrm{H}) .{ }^{13} \mathrm{C} \mathrm{NMR}$ (126 MHz, acetone-d6): $\delta 143.57,138.71,132.52,129.28,129.23,129.08,128.34$, 127.70, 126.79, 126.37, 70.81, 42.27, 39.89, 32.71. HRMS (FAB $\left.{ }^{+}\right)$: Calculated for $\mathrm{C}_{18} \mathrm{H}_{20} \mathrm{O}+\mathrm{H}^{+}: 253.1592$; Found: 253.1592 .

(4E)-5-(2-methoxyphenyl)-2-methylpent-4-en-2-ol (1 m).

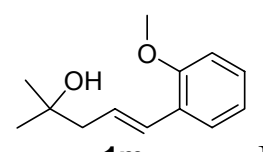

$1 \mathrm{~m} \quad$ Following the above procedure, with acetone and 2-iodoanisole in the presence of (allylPdCl $)_{2}$ as catalyst, $\mathbf{1 m}$ was afforded as a clear oil in 45\% yield (196 mg). ${ }^{1} \mathrm{H}$ NMR (500 MHz, acetone-d6): $\delta 7.47$ (dd, $\left.J=7.6,1.5 \mathrm{~Hz}, 1 \mathrm{H}\right) ; 7.20-7.16(\mathrm{~m}, 1$ $\mathrm{H}) ; 6.94(\mathrm{~d}, J=7.9 \mathrm{~Hz}, 1 \mathrm{H}) ; 6.88(\mathrm{t}, J=7.5 \mathrm{~Hz}, 1 \mathrm{H}) ; 6.71(\mathrm{~d}, J=15.9 \mathrm{~Hz}, 1 \mathrm{H}) ; 6.34$ (dt, $J=16.0,7.5 \mathrm{~Hz}, 1 \mathrm{H}) ; 3.82(\mathrm{~s}, 3 \mathrm{H}) ; 3.38(\mathrm{~s}, 1 \mathrm{H}, \mathrm{OH}) ; 2.35(\mathrm{~d}, J=7.4 \mathrm{~Hz}, 2 \mathrm{H})$; 1.19 (s, $6 \mathrm{H}) .{ }^{13} \mathrm{C}$ NMR (126 MHz, acetone-d6): $\delta 157.24,128.87,128.23,127.52$, 127.45, 126.93, 121.30, 111.76, 70.50, 55.73, 48.83, 29.69. HRMS (FAB ${ }^{+}$): Calculated for $\mathrm{C}_{13} \mathrm{H}_{18} \mathrm{O}_{2}+\mathrm{H}^{+}: 207.1385$; Found: 207.1385 .

1-[(2E)-3-(2-methoxyphenyl)prop-2-en-1-yl]cyclohexanol (1n). 


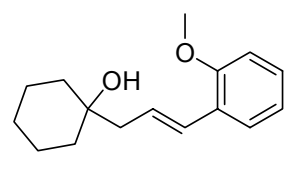

1n Following the above procedure, with cyclohexanone and 2-iodoanisole in the presence of $(\text { allylPdCl })_{2}$ as catalyst, 1 n was afforded as a clear oil in $41 \%$ yield (214 mg). ${ }^{1} \mathrm{H}$ NMR (500 MHz, acetone- $\left.d 6\right): \delta 7.47(\mathrm{dd}, J=7.6,1.5 \mathrm{~Hz}, 1 \mathrm{H}) ; 7.18-7.16(\mathrm{~m}, 1$ $\mathrm{H}) ; 6.94(\mathrm{~d}, J=8.2 \mathrm{~Hz}, 1 \mathrm{H}) ; 6.87(\mathrm{t}, J=7.4 \mathrm{~Hz}, 1 \mathrm{H}) ; 6.70(\mathrm{~d}, J=16.0 \mathrm{~Hz}, 1 \mathrm{H}) ; 6.36$ $(\mathrm{dt}, J=16.0,7.5 \mathrm{~Hz}, 1 \mathrm{H}) ; 3.82(\mathrm{~s}, 3 \mathrm{H}) ; 3.15(\mathrm{~s}, 1 \mathrm{H}, \mathrm{OH}) ; 2.33(\mathrm{dd}, J=7.5,1.3 \mathrm{~Hz}, 2$ $\mathrm{H})$; $1.69-1.61(\mathrm{~m}, 2 \mathrm{H}) ; 1.56-1.40(\mathrm{~m}, 7 \mathrm{H}) ; 1.27-1.19(\mathrm{~m}, 1 \mathrm{H}) .{ }^{13} \mathrm{C} \mathrm{NMR}(126 \mathrm{MHz}$, acetone-d6): $\delta 157.19,128.83,127.68,127.51,126.91,121.31,111.75,71.34,55.76$, 47.85, 38.22, 26.69, 22.85. HRMS $\left(\mathrm{FAB}^{+}\right)$: Calculated for $\mathrm{C}_{16} \mathrm{H}_{22} \mathrm{O}_{2}+\mathrm{K}^{+}$: 285.1257; Found: 285.1257.

(5E)-6-(2-methoxyphenyl)-1-phenylhex-5-en-3-ol (10).

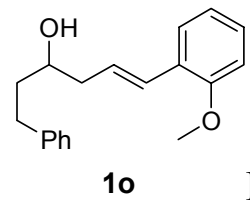
in the presence of (allylPdCl $)_{2}$ as catalyst, 10 was afforded as a clear oil in $71 \%$ yield (421 mg). ${ }^{1} \mathrm{H}$ NMR (500 MHz, acetone- $\left.d 6\right): \delta 7.44(\mathrm{dd}, J=7.7,1.5 \mathrm{~Hz}, 1 \mathrm{H}) ; 7.26-7.12$ $(\mathrm{m}, 6 \mathrm{H}) ; 6.94(\mathrm{~d}, J=7.8 \mathrm{~Hz}, 1 \mathrm{H}) ; 6.87(\mathrm{t}, J=7.4 \mathrm{~Hz}, 1 \mathrm{H}) ; 6.73(\mathrm{~d}, J=16.0 \mathrm{~Hz}, 1 \mathrm{H})$; $6.31(\mathrm{dt}, J=16.0,7.3 \mathrm{~Hz}, 1 \mathrm{H}) ; 3.81(\mathrm{~s}, 3 \mathrm{H}) ; 3.74-3.68(\mathrm{~m}, 2 \mathrm{H}, \mathrm{C} \underline{\mathrm{HOH}}) ; 2.88-2.64$ (m, $2 \mathrm{H})$; 2.42-2.36 (m, $2 \mathrm{H}) ; 1.85-1.69(\mathrm{~m}, 2 \mathrm{H}) .{ }^{13} \mathrm{C}$ NMR (126 MHz, acetone- $\left.d 6\right)$ : $\delta 157.24,143.62,129.27,129.10,128.91,128.48,127.40,127.17,126.96,126.39,121.32$, 111.75, 70.97, 55.72, 42.76, 39.89, 32.75. HRMS (FAB ${ }^{+}$): Calculated for $\mathrm{C}_{19} \mathrm{H}_{22} \mathrm{O}_{2}+\mathrm{K}^{+}$: 321.1258; Found: 321.1257. 

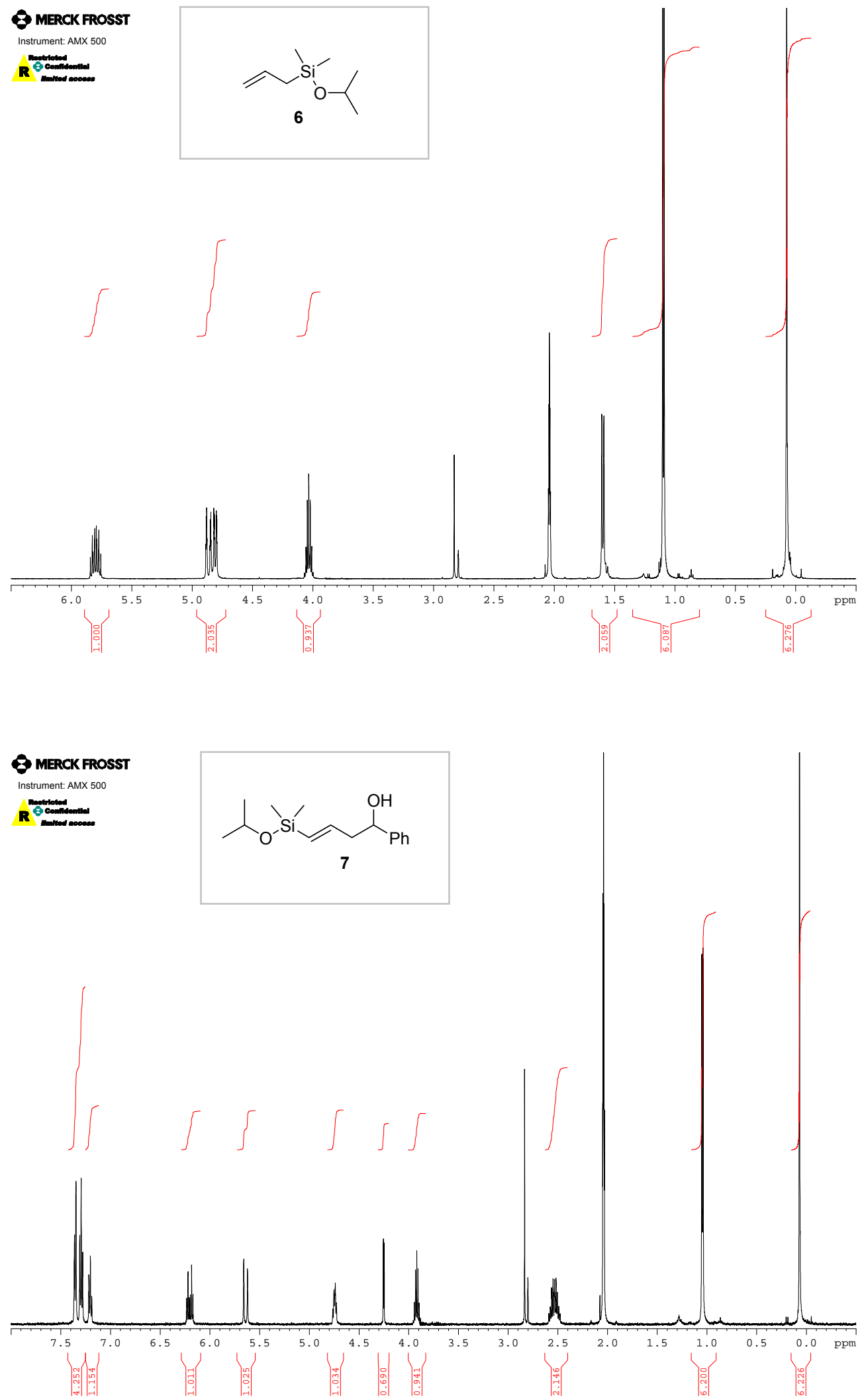
Support Information for Manuscript OL048892L, by L. Li ${ }^{*}$ and N. Navasero 26 pages

8. MERCK FROSST Instrument: AMX 500

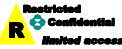
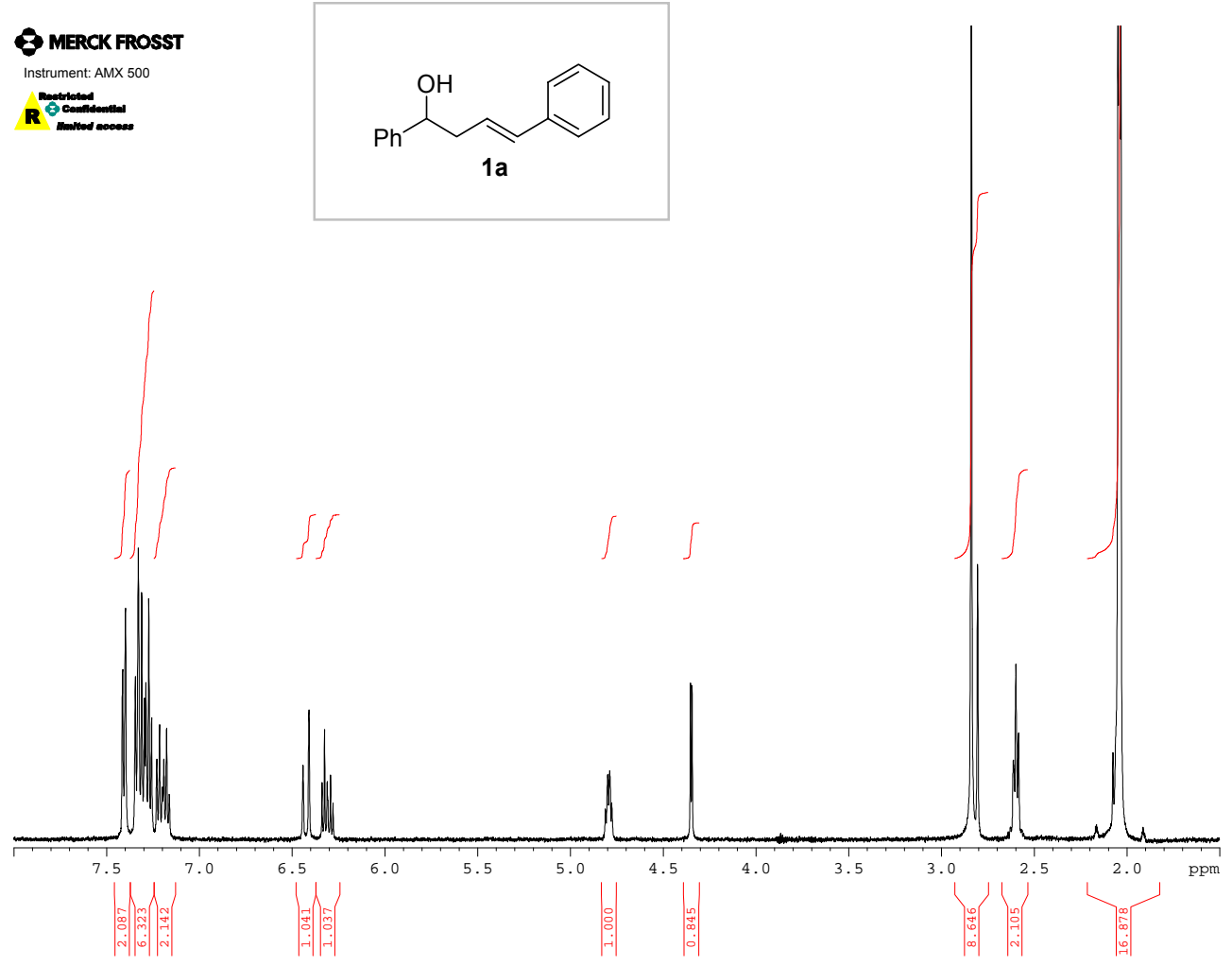

8. MERCK FROSST Instrument: AMX 500

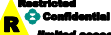
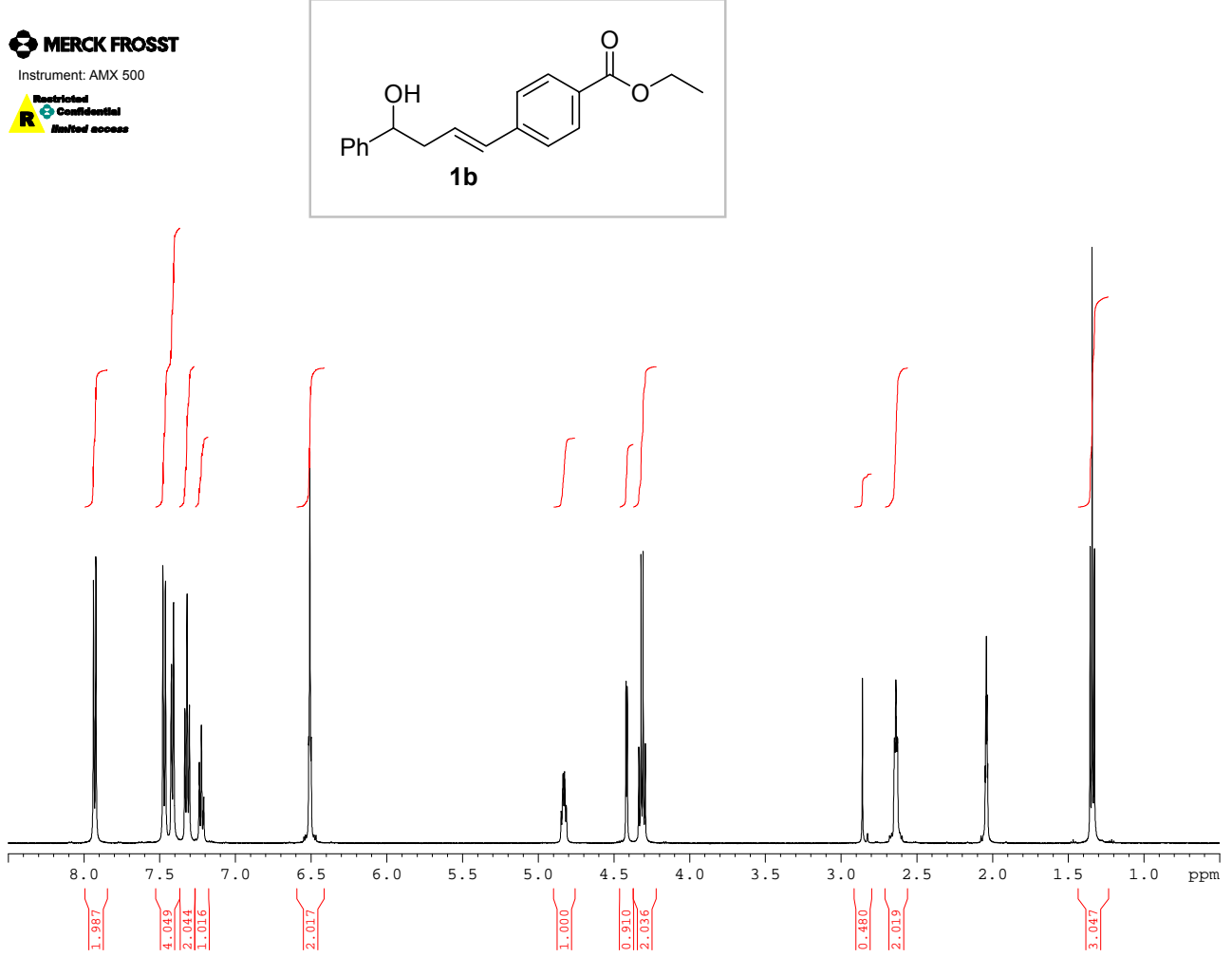

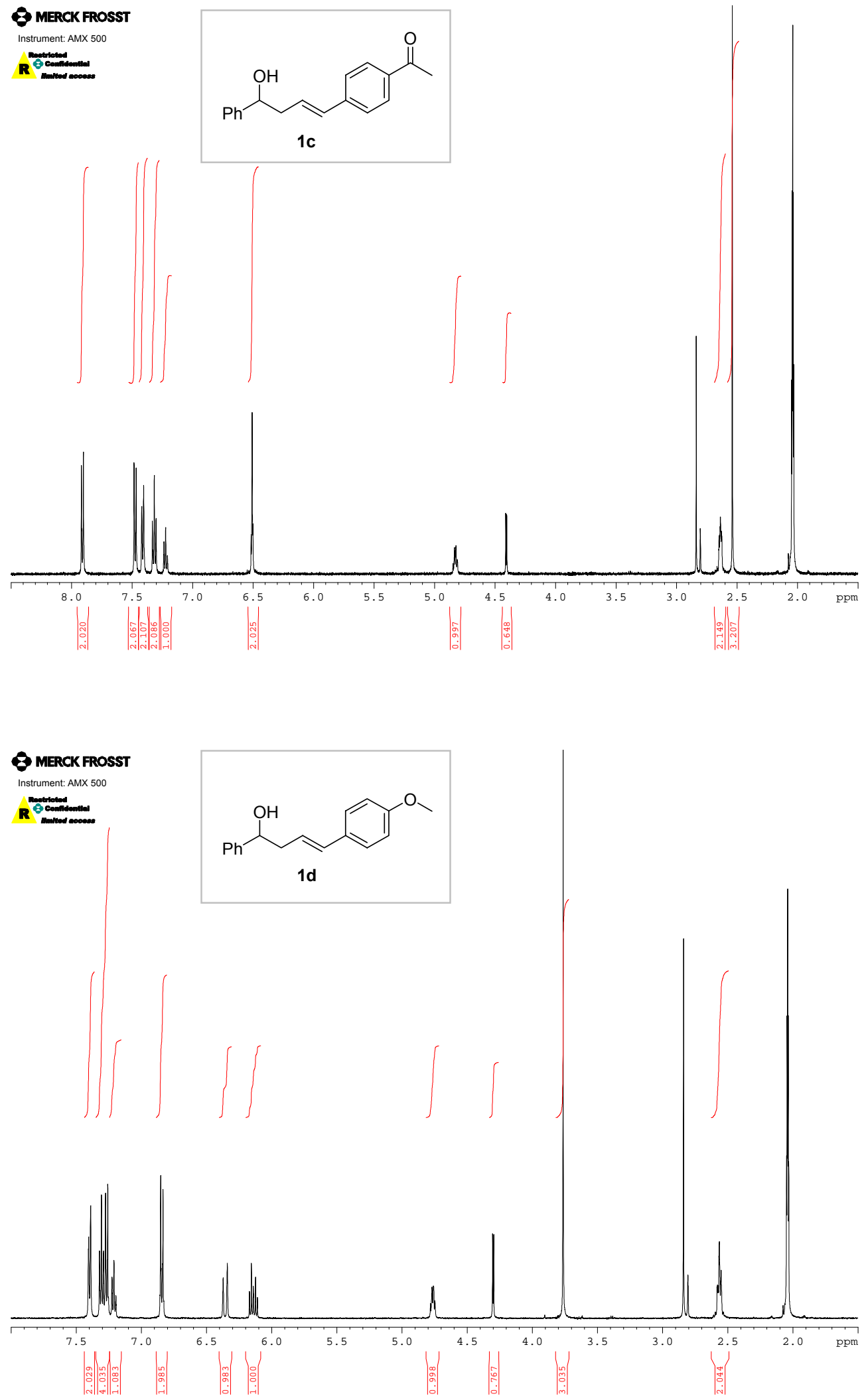

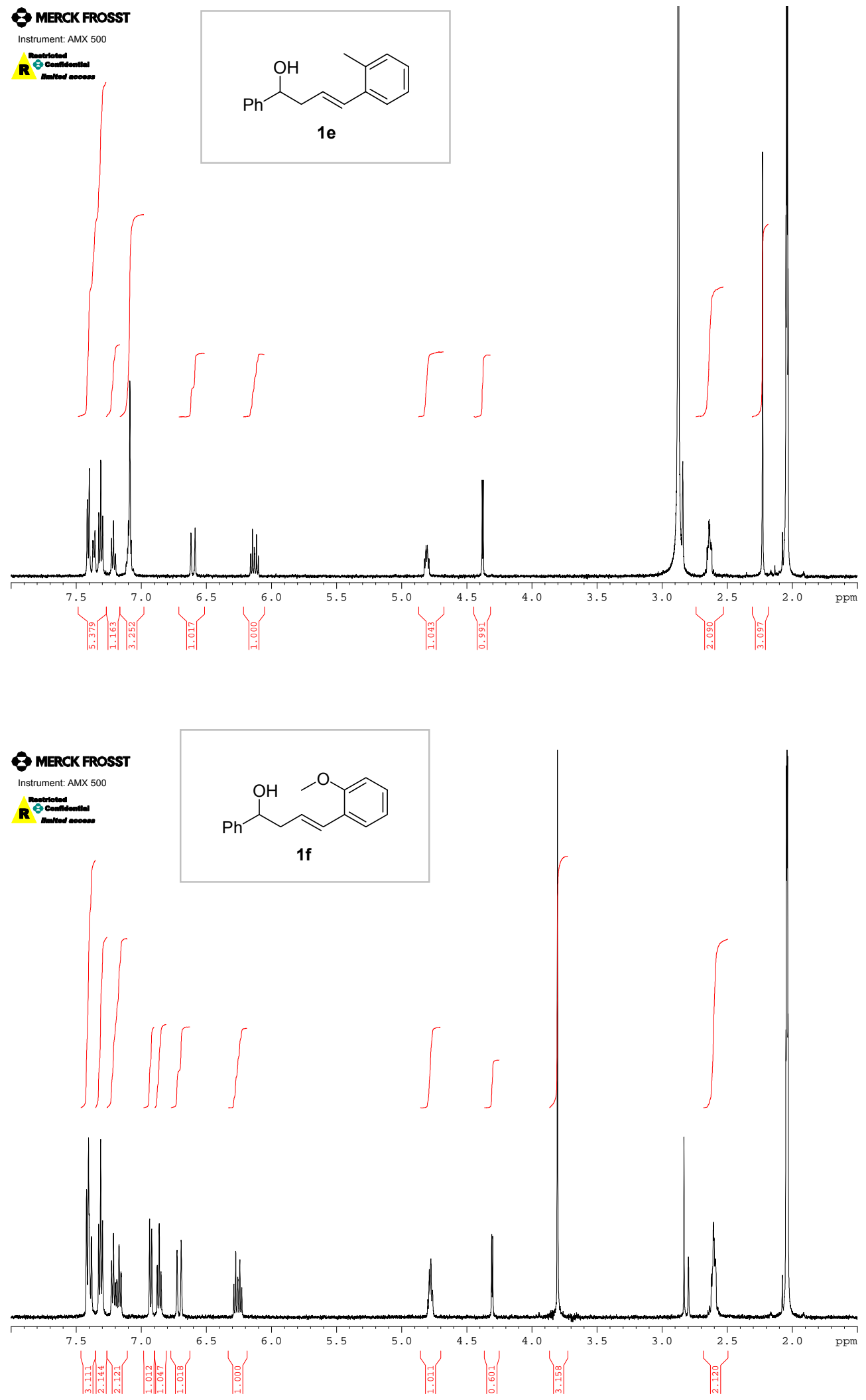

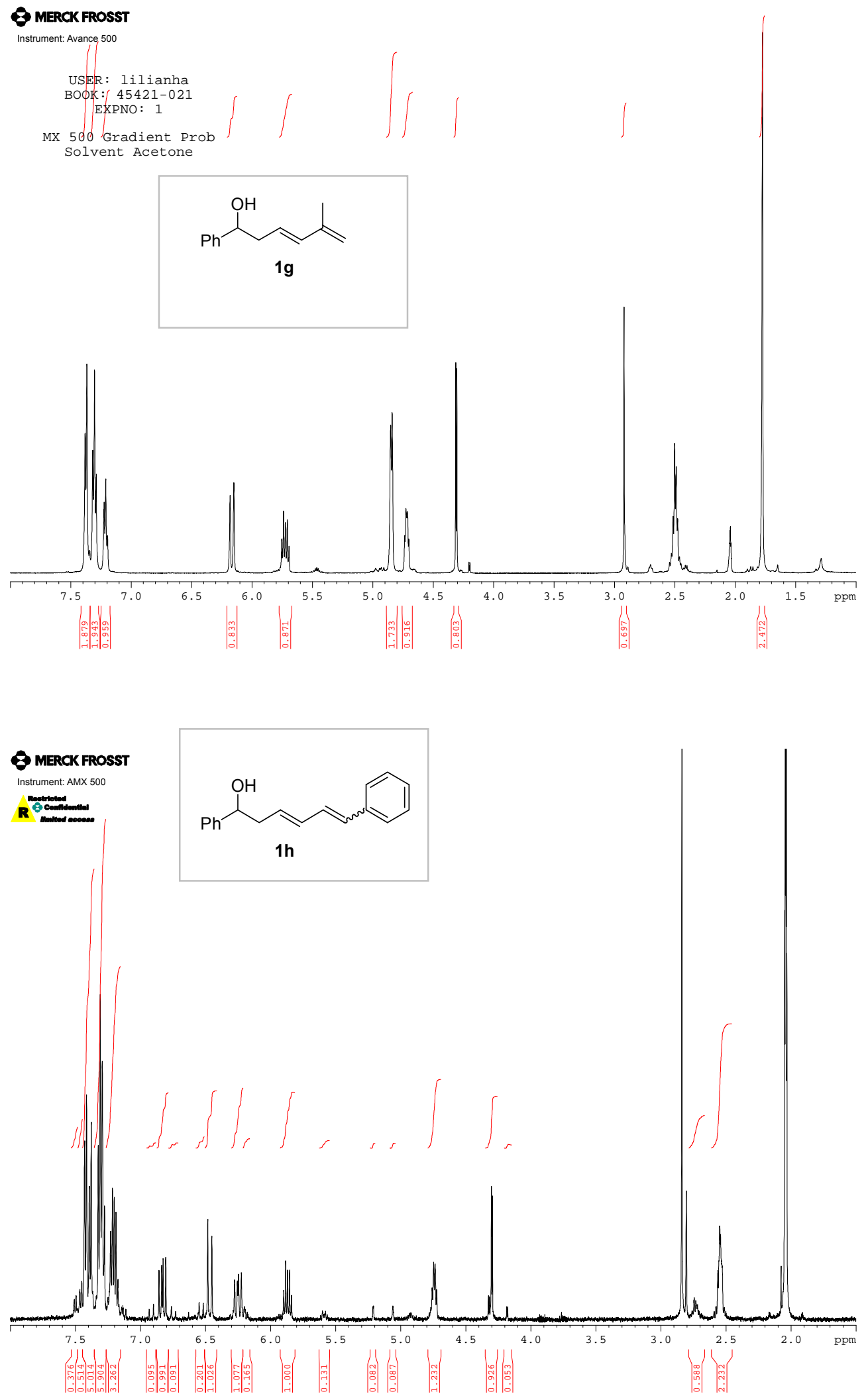

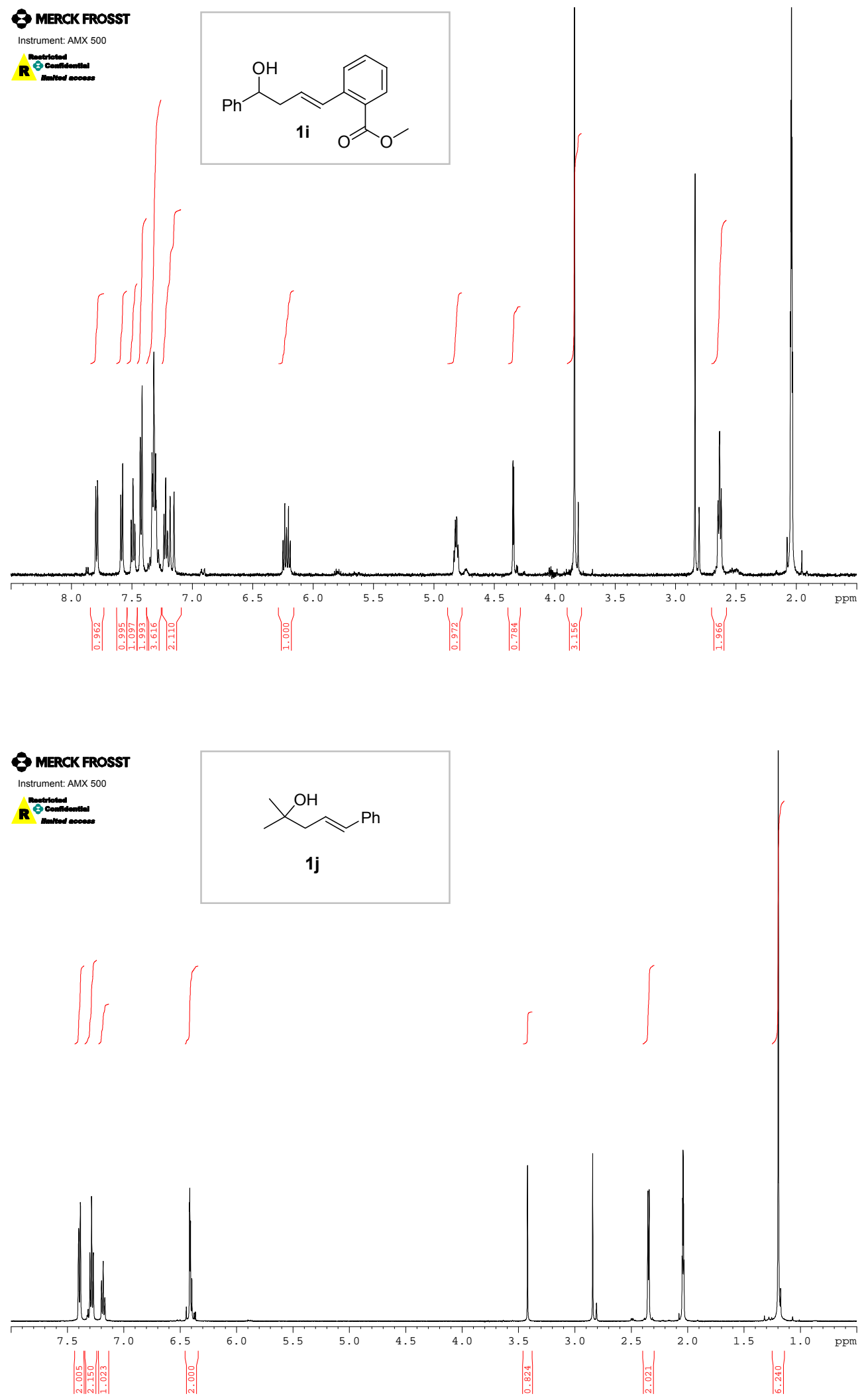

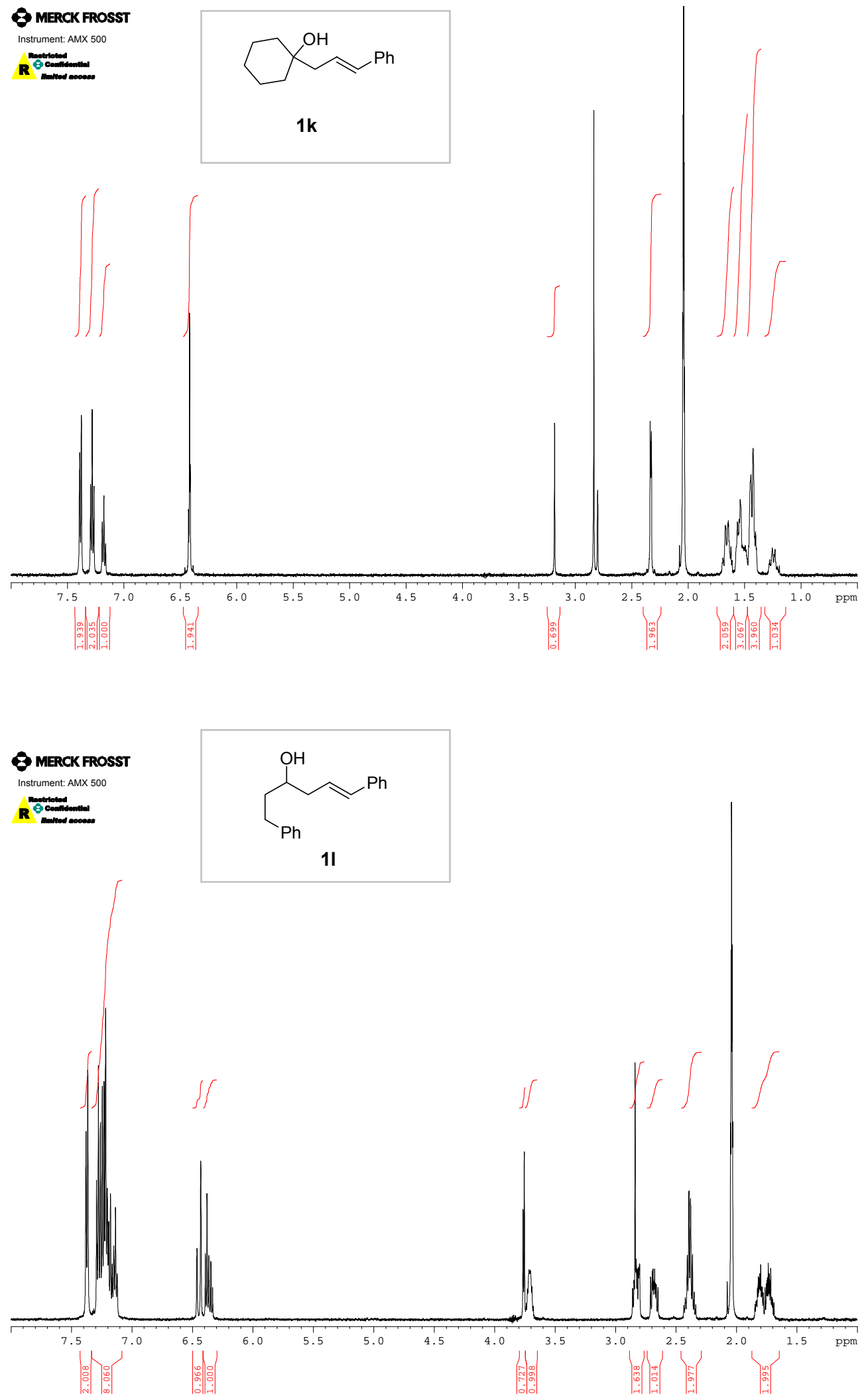

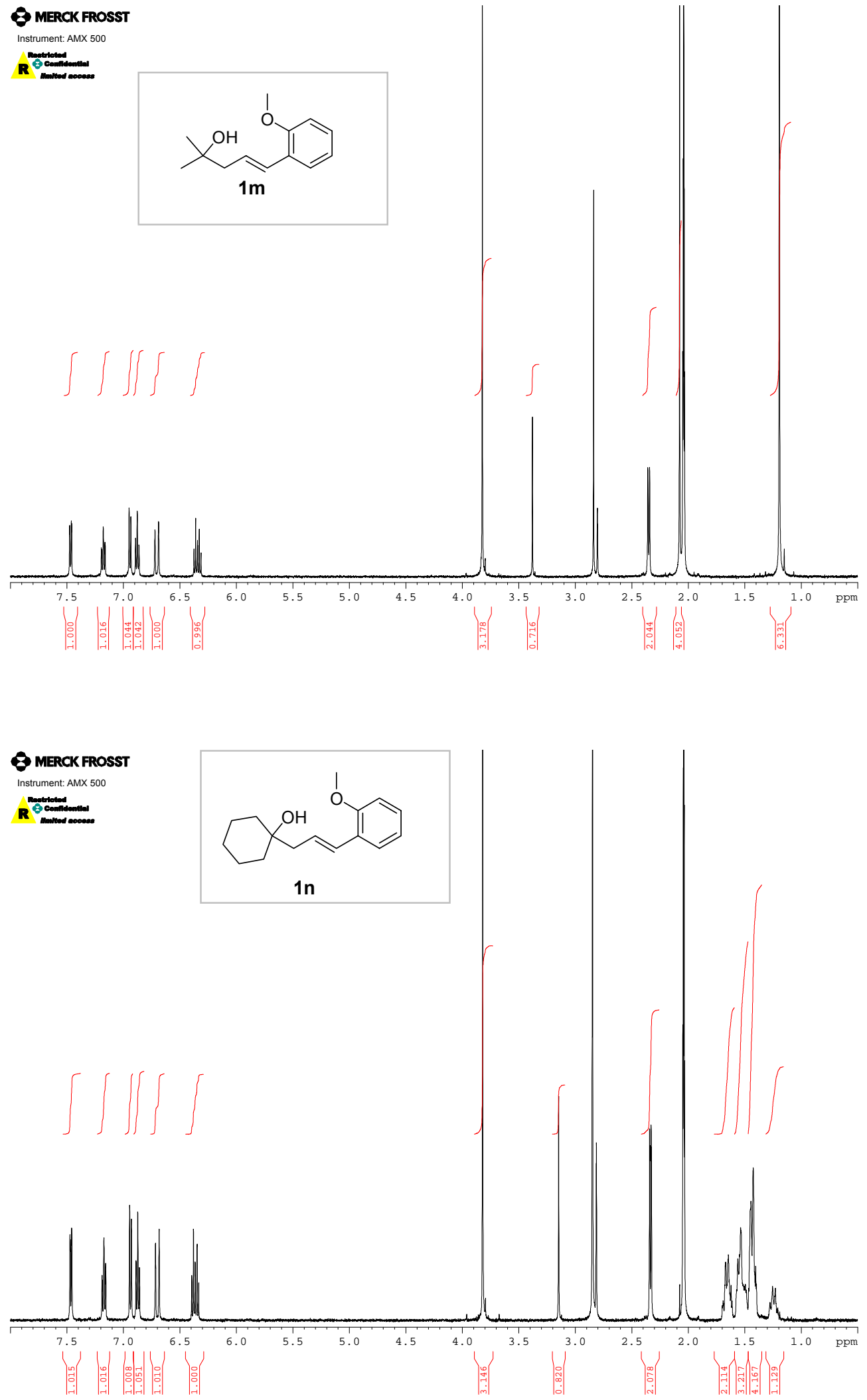


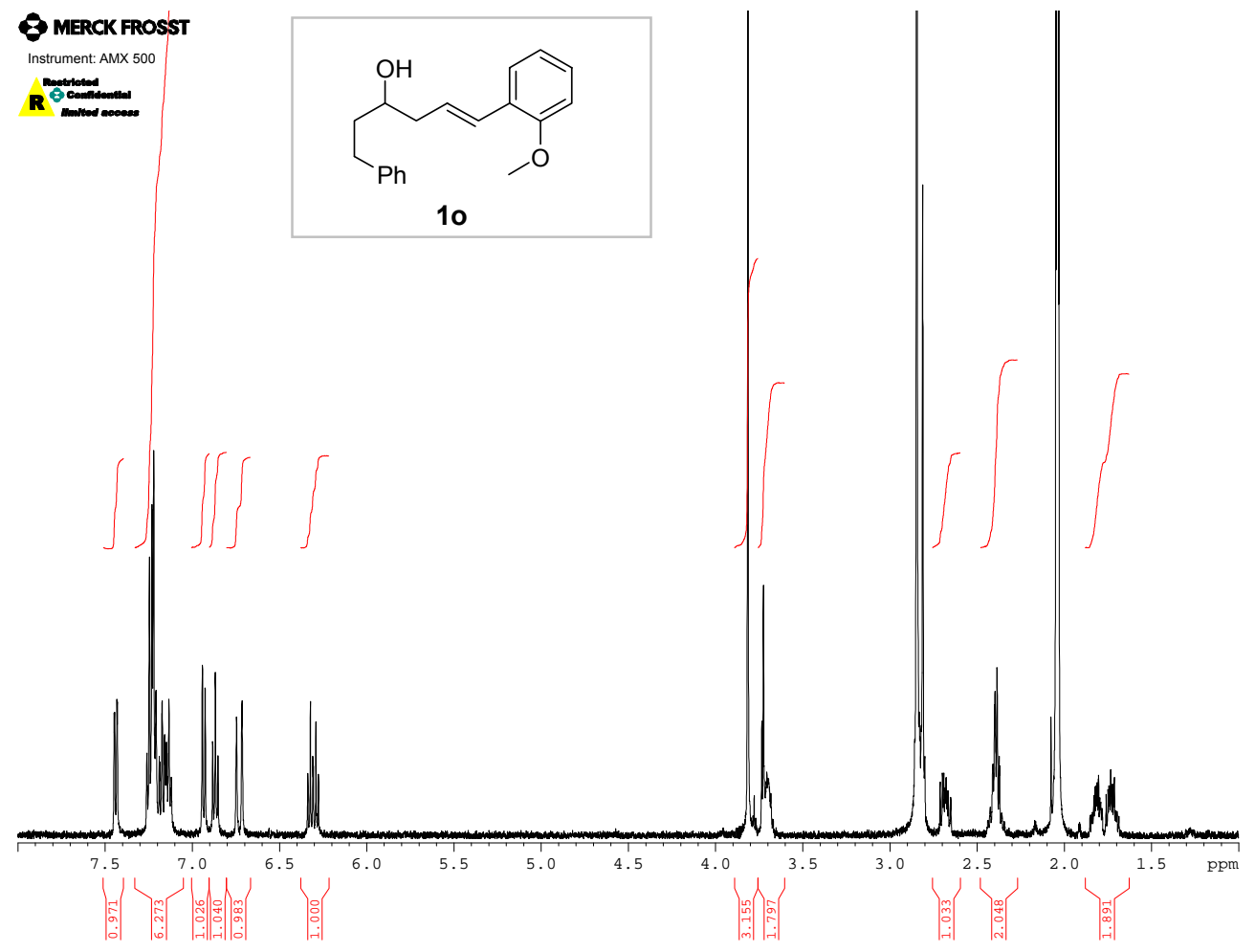



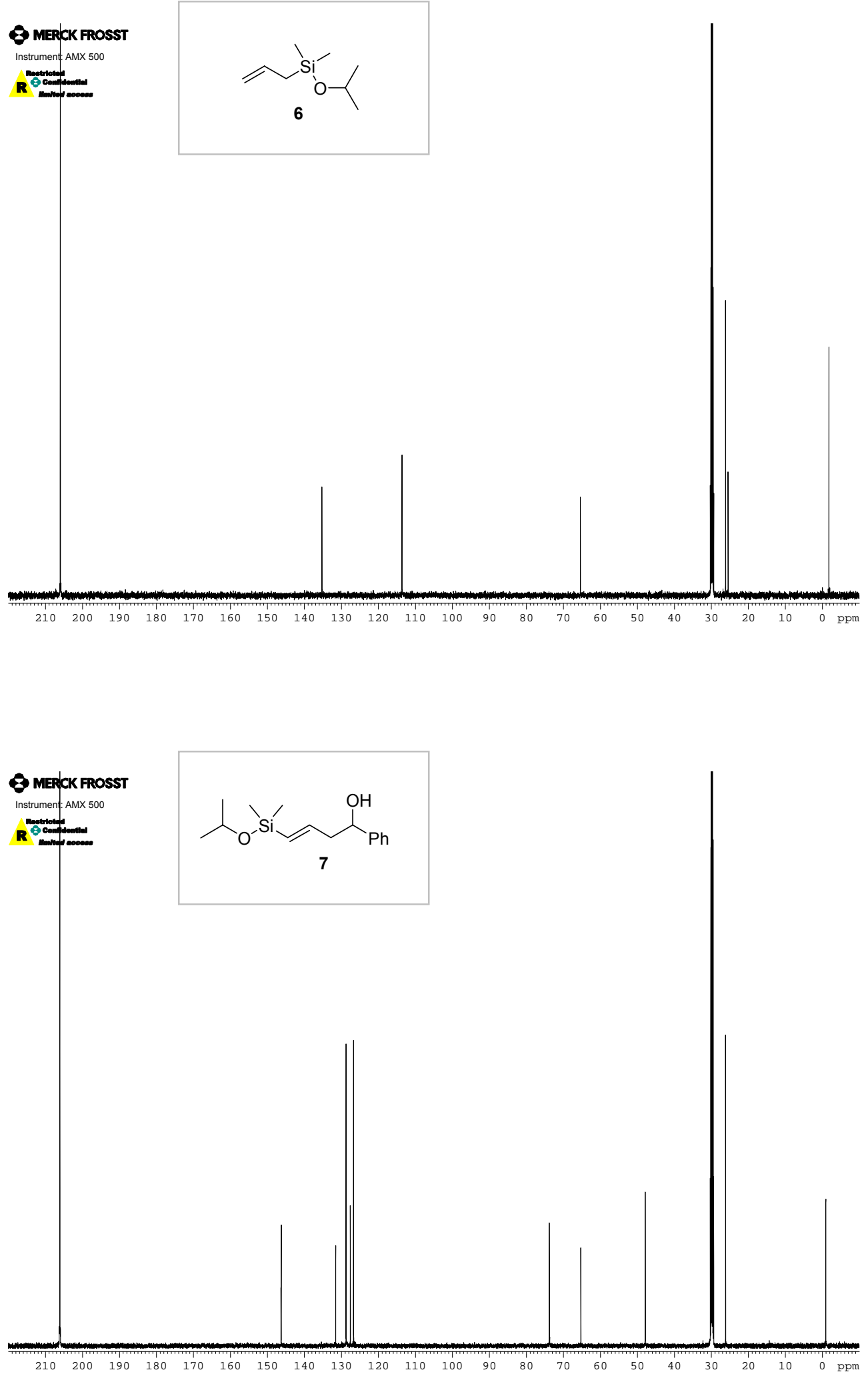

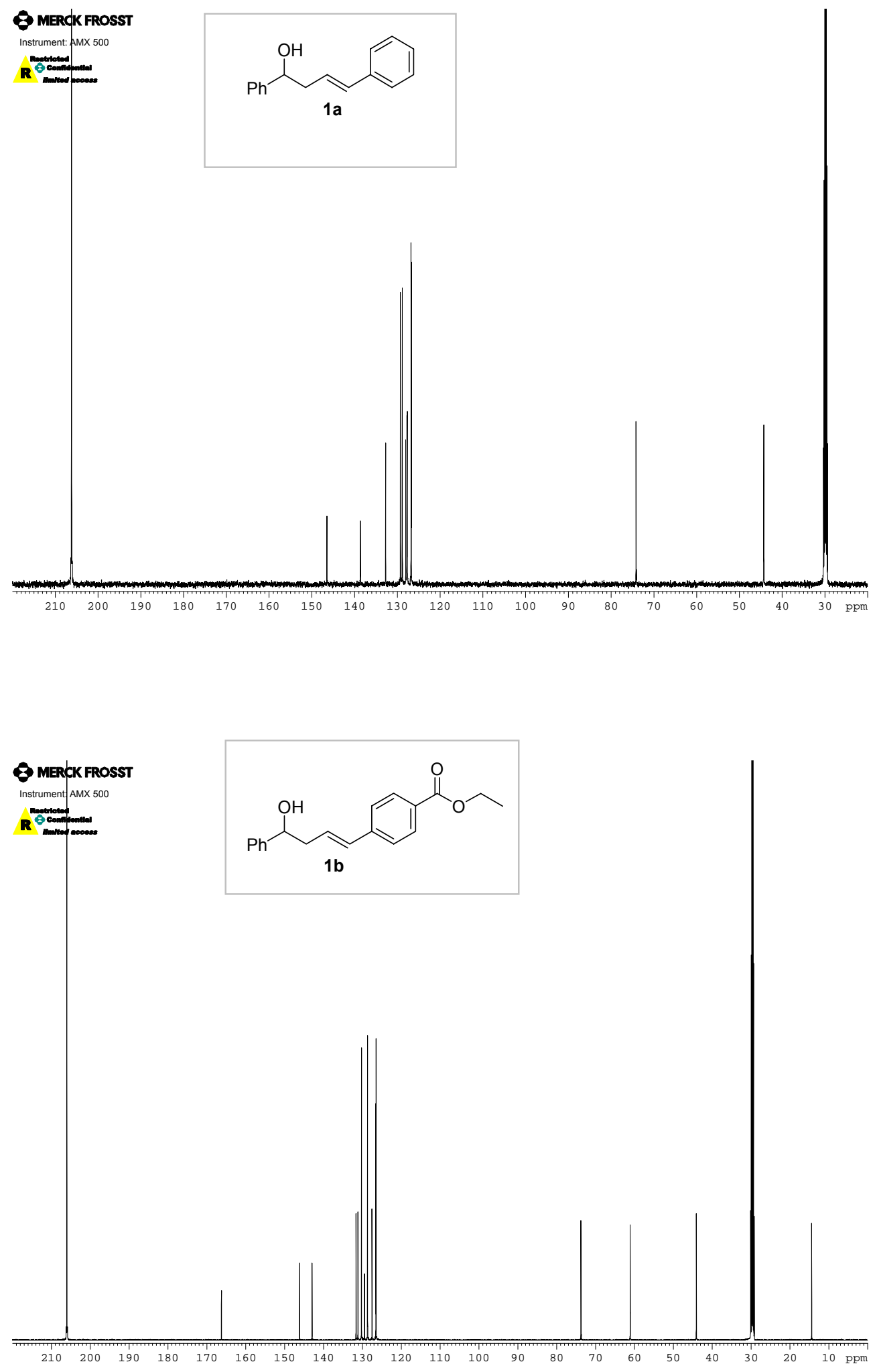

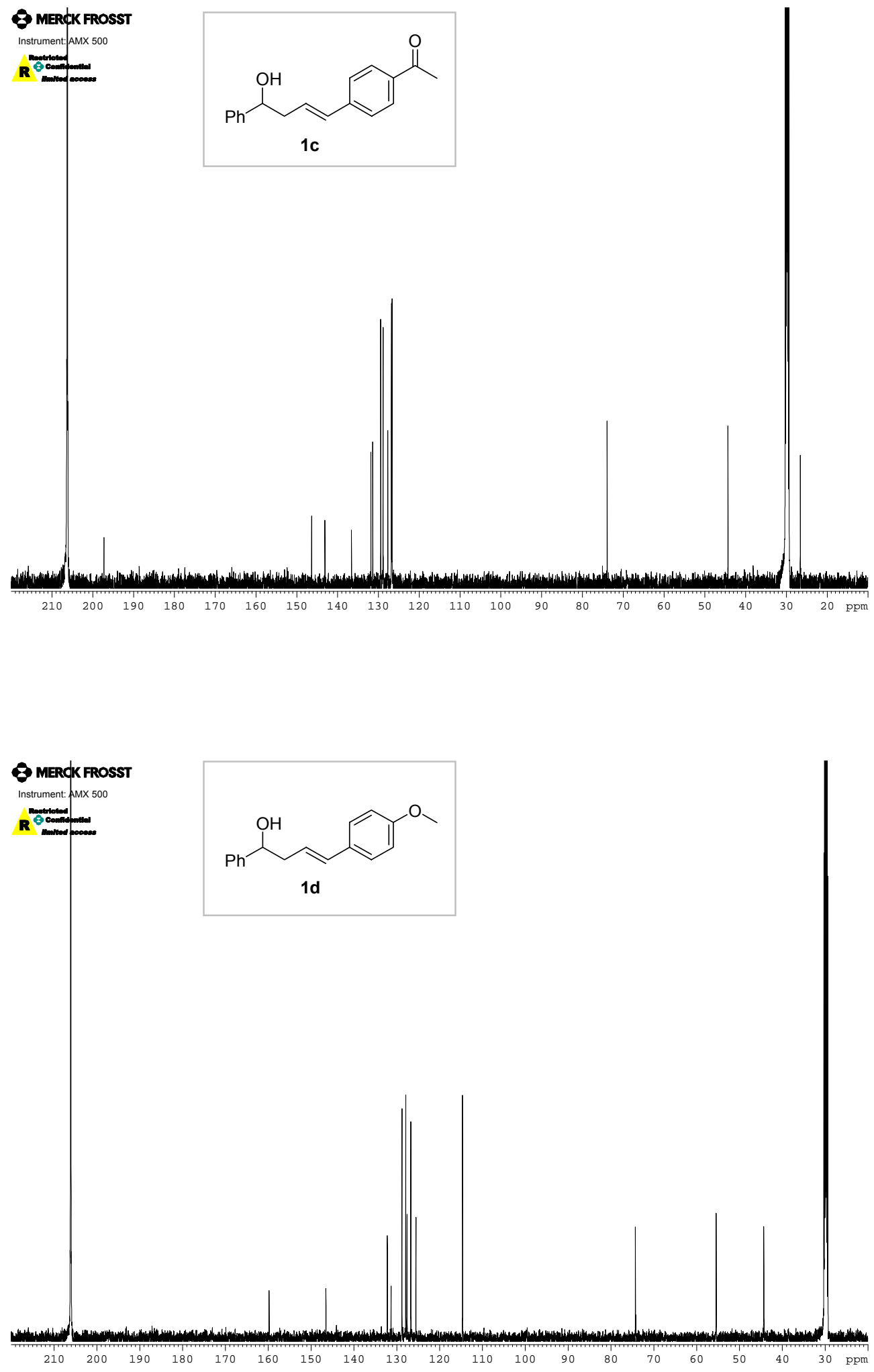

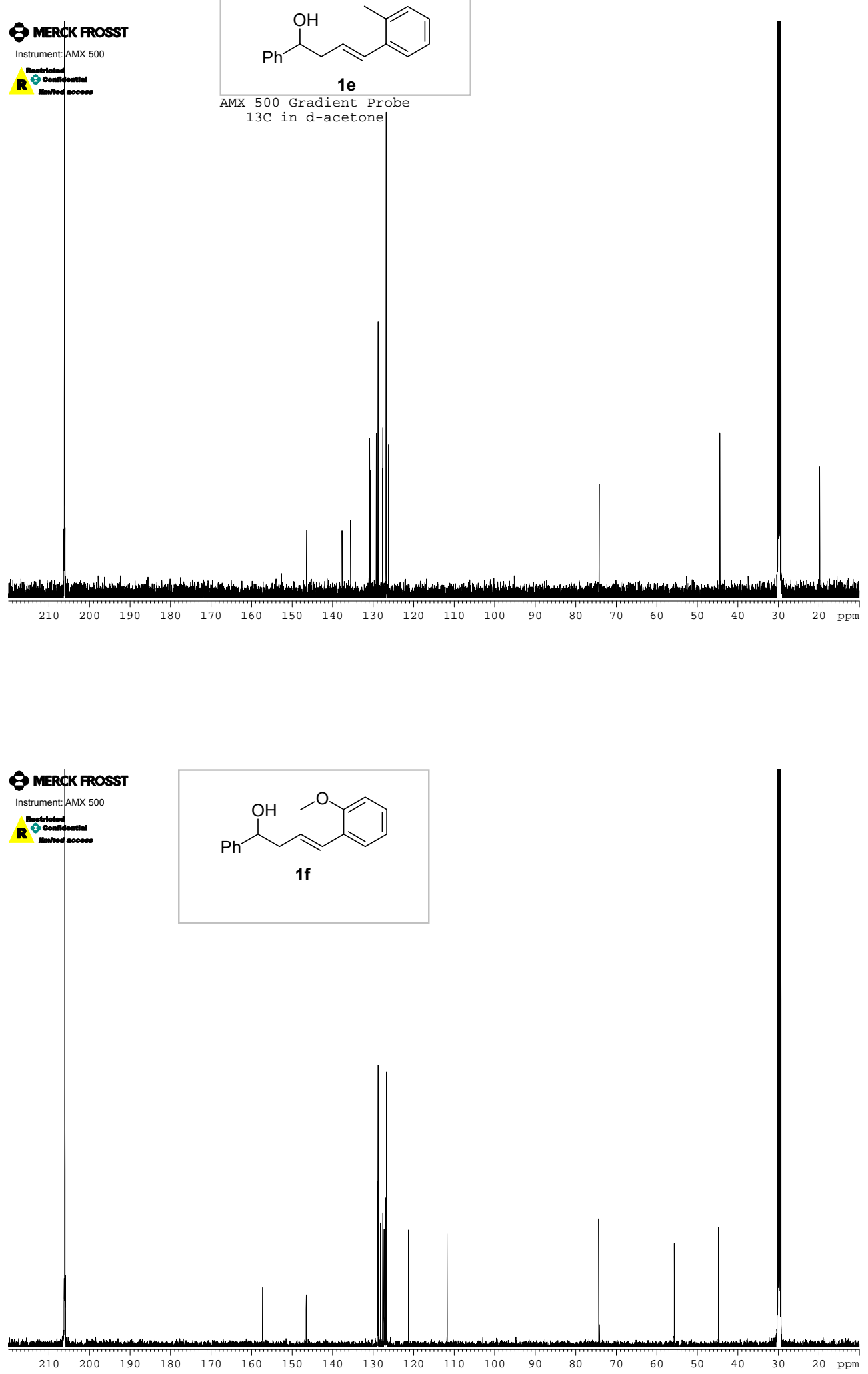

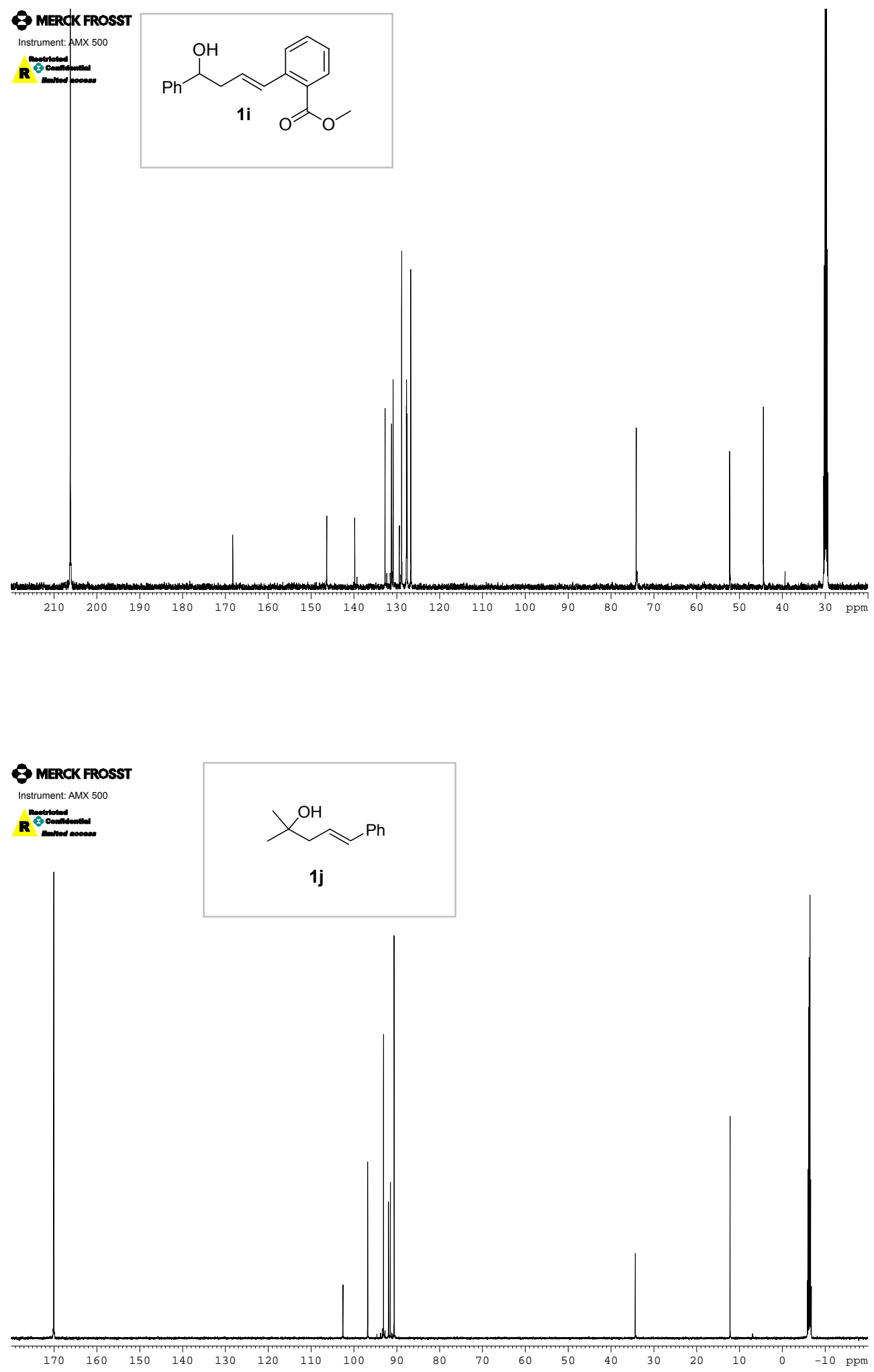

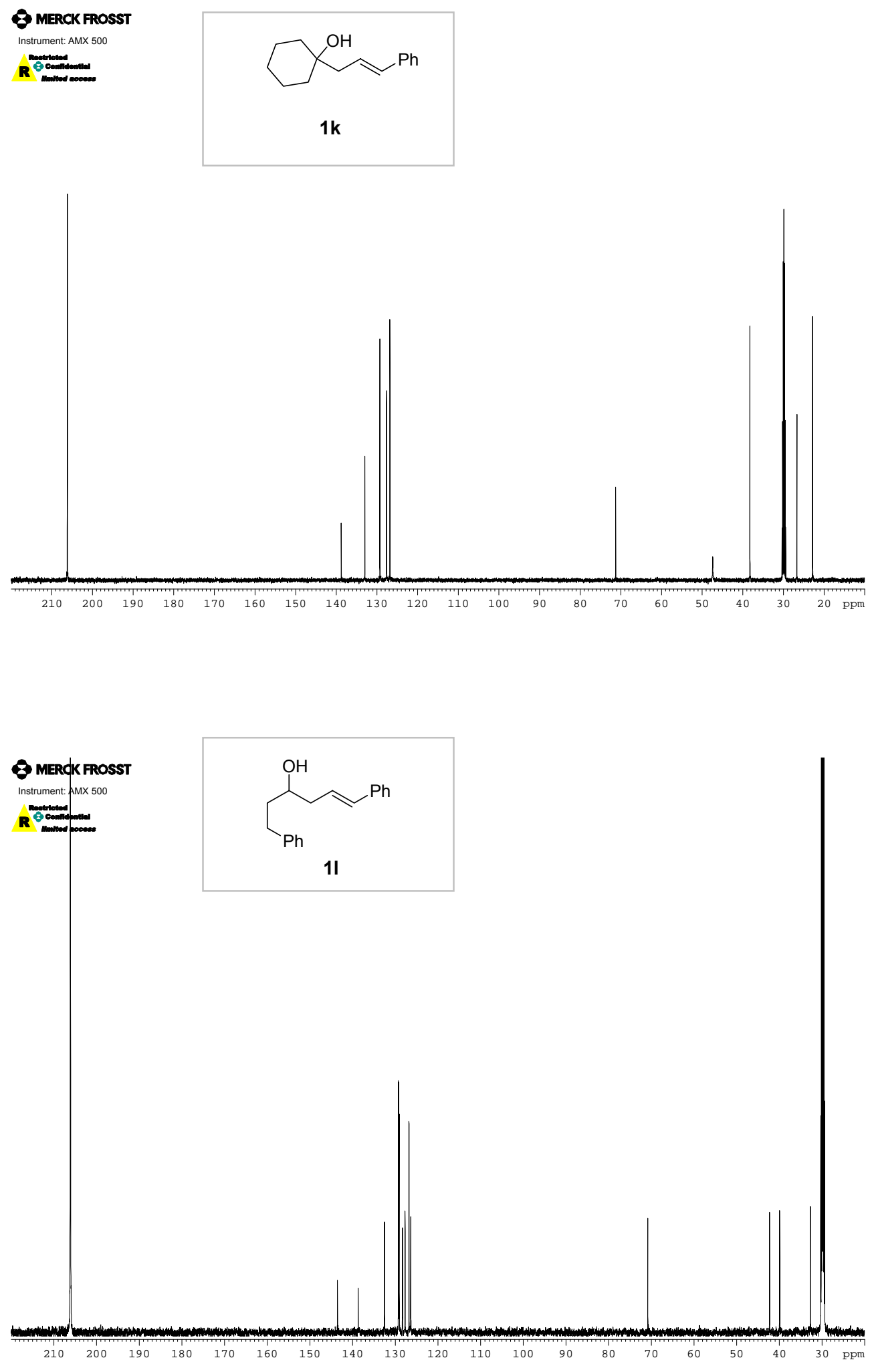

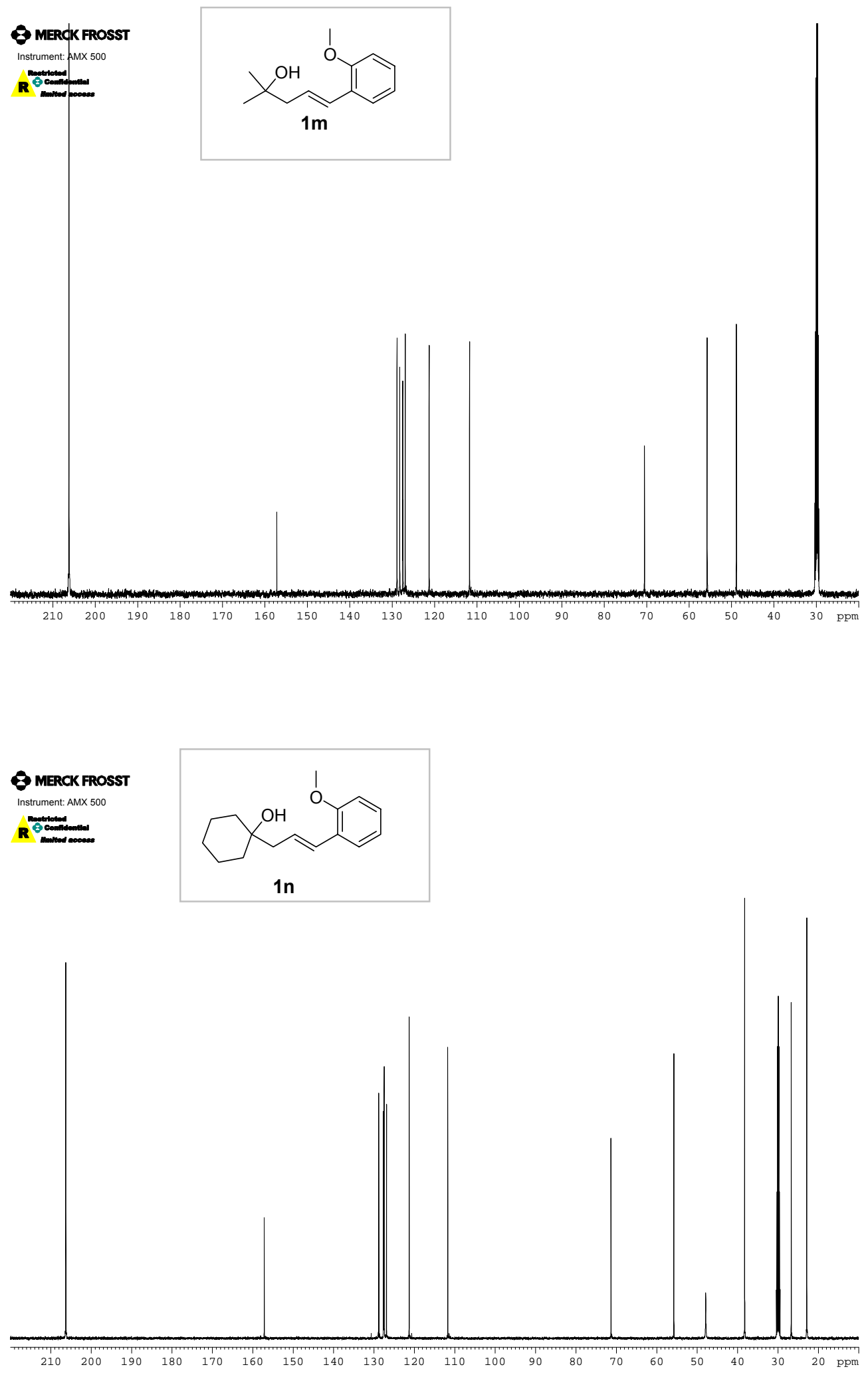
Support Information for Manuscript OL048892L, by L. Li ${ }^{*}$ and N. Navasero 26 pages

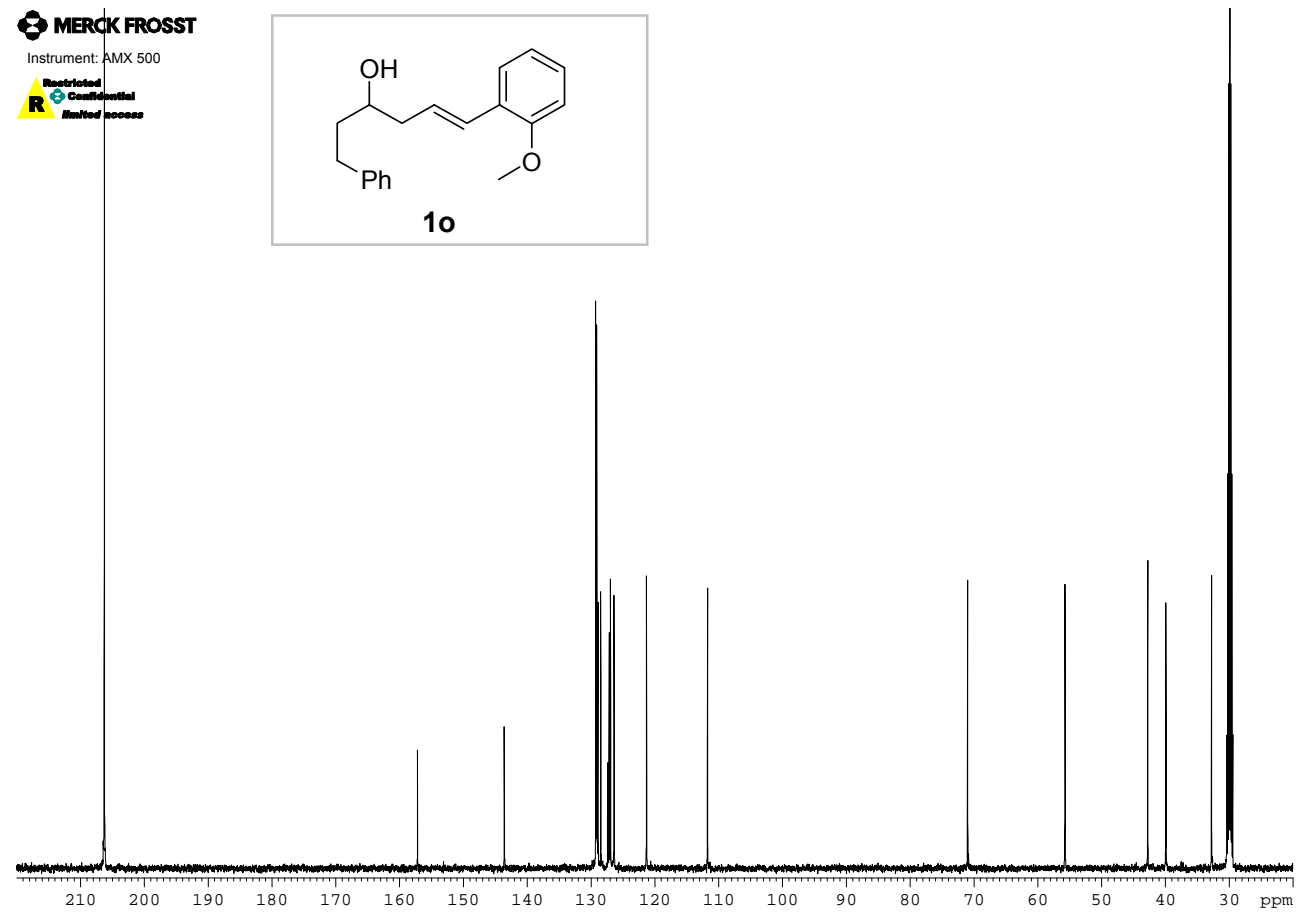

